\title{
Evaluation of computational models of infant language development against robust empirical data from meta-analyses: what, why, and how?
}

\author{
María Andrea Cruz Blandón*a ${ }^{* a}$ Alejandrina Cristia ${ }^{\dagger b}$, and Okko Räsänen ${ }^{\ddagger a}$ \\ ${ }^{a}$ Unit of Computing Sciences, Faculty of Information Technology and \\ Communication Sciences, Tampere University, Finland \\ ${ }^{\mathrm{b}}$ Laboratoire de Sciences Cognitives et Psycholinguistique, Département d'études \\ cognitives, ENS, EHESS, CNRS, PSL University, France
}

Draft version 1.0, December 13, 2021

\begin{abstract}
Computational models of child language development can help us understand the cognitive underpinnings of the language learning process. One advantage of computational modeling is that is has the potential to address multiple aspects of language learning within a single learning architecture. If successful, such integrated models would help to pave the way for a more comprehensive and mechanistic understanding of language development. However, in order to develop more accurate, holistic, and hence impactful models of infant language learning, the research on models also requires model evaluation practices that allow comparison of model behavior to empirical data from infants across a range of language capabilities. Moreover, there is a need for practices that can compare developmental trajectories of infants to those of models as a function of language experience. The present study aims to take the first steps to address these needs. More specifically, we will introduce the concept of comparing models with largescale cumulative empirical data from infants, as quantified by meta-analyses conducted across a large number of individual behavioral studies. We start by formalizing the connection between measurable model and human behavior, and then present a basic conceptual framework for meta-analytic evaluation of computational models together with basic guidelines intended as a starting point for later work in this direction. We exemplify the meta-analytic model evaluation approach with two modeling experiments on infant-directed speech preference and native/nonnative vowel discrimination. We also discuss the advantages, challenges, and potential future directions of meta-analytic evaluation practices.
\end{abstract}

Keywords: computational modeling, child language development, meta-analysis, model evaluation, language acquisition

\footnotetext{
*maria.cruzblandon@tuni.fi

†alejandrina.cristia@ens.fr

‡okko.rasanen@tuni.fi. Corresponding author.
} 


\section{Introduction}

How children learn their native language has long puzzled researchers across disciplines. Countless behavioral studies and empirical observations have been used as a means to understand the underpinnings of language development. This research has produced many breakthroughs and insights on various language and learning-related phenomena in infants and older children. However, we are still lacking a mechanistic, accurate, and comprehensive model of the learning process that would explain how the bits and pieces of accumulated understanding fit together. This is largely because language and its acquisition are extremely complex phenomena, consisting of a dynamic interplay of linguistic, sociocultural, developmental, genetic, and other potential factors that together give rise to the language capabilities we define, observe, and measure in children. For example, one of the continual challenges is the lack of an integrated model of how phonetic and phonemic category acquisition, word learning, and emerging syntactic and semantic skills interact and support each other at different stages of the developmental timeline (see, e.g., Lewis et al., 2016 or Dupoux, 2018, for a discussion). Studying such interactions across multiple levels of language is very challenging with controlled behavioral experiments.

In parallel to empirical research, there is a long tradition of developing computational models for language development and speech perception in order to have more formal accounts of language processing in the cognitive system and to test theories in practice. Other work has laid out the promise of computational modeling in particular, and formalization in general, to make progress in psychological theorizing (e.g., Fried, 2020; Guest and Martin, 2021; Robinaugh et al., 2021), and thus we will not repeat those arguments. ${ }^{1}$ In addition to providing fuller accounts of a process, models can also act as learnability proofs for various linguistic phenomena, such as demonstrating what type of linguistic representations and skills are achievable using a given set of assumptions, learning mechanisms, and data to learn from (see also Alishahi, 2010; Räsänen, 2012). Since computational models can, and must, define all the processing steps from low-level inputs (e.g., acoustic speech) to high-level behavior (e.g., observable language skills), one of the big promises in computational modeling is its potential to integrate several "sub-processes" of language learning into unified testable frameworks. As an example, a model combining phonetic, lexical, semantic, and syntactic learning into a single model architecture would enable systematic and reproducible investigation of the mechanisms involved and their interactions, helping us to understand what can lead to human-like language learning behavior across these levels of analysis (Dupoux, 2018). Given the recent advances in machine learning, availability of data, and availability of open source code and pre-trained model components, major steps have been made on this path from models tackling individual language phenomena (e.g., word segmentation or phonetic category learning) towards more comprehensive approaches attempting to learn at multiple levels (e.g., Alishahi et al., 2021; Nguyen et al., 2020).

Besides the computational research working on human models of language learning, there is an ongoing trend in machine learning and AI research towards increasingly unsupervised and multimodal learning algorithms (e.g., Harwath et al., 2019; Oord et al., 2018). Such algorithms can learn structural patterns and cross-modal dependencies autonomously from low-level sensory data, being in contrast to classical supervised training and the effort required to deploy such system to novel domains. This narrows the methodological gap between application-oriented machine learning systems and computational language development research, as both (primarily) operate on the basic idea of unsupervised pattern discovery from environmental input. Some studies have also started to look at parallels between machine learning system behavior and human (adult) speech perception data (e.g., Havard et al., 2019; Magnuson et al., 2020; Scholten et al., 2021).

However, in order for us to better understand how infants learn their native language(s), a critical

\footnotetext{
${ }^{1}$ For further information about why and how to go from verbal to formalized theories, see Borsboom et al. (2021).
} 
component is the evaluation of the computational models (and machine learning algorithms) with respect to infant data on language learning. ${ }^{2}$ More specifically, if we wish to model infant learning, our models should be able to replicate the developmental trajectories of infant learners (see also Alishahi, 2010 and Dupoux, 2018, for a discussion). In contrast, evaluation in terms of model compatibility with the way how linguistic theory describes (or prescribes) the units and structure of spoken language can only act as a proxy for infant language skills. The reason behind this is that the psychological role of linguistic representations in different stages of language development is still an active area of research, and furthermore the development of language skills does not necessarily follow linear trajectories. In fact, there is a theoretical possibility that linguisticallymotivated model evaluation methods may even lead researchers astray, as models with a good fit to human data might not be those that provide the best performance scores in terms of learning and representing various linguistic structures from speech input (e.g., Larsen et al., 2017). Hence, comparison of computational models against actual infant data should be a high priority for those willing to understand human learning processes with computational means.

As simple as evaluation with respect to human data sounds, the process comes with many practical and conceptual challenges (see also online supplementary material in Schatz et al., 2021). How can one compare outputs and internal representations of programmed algorithms running inside a computer against behavioral data such as measurements of infant gaze or EEG responses from laboratory experiments? How does one ensure that the model does not overfit ${ }^{3}$ to the data of interest, given that typical behavioral data from a study is relatively low-dimensional whereas models tend to come with countless design choices (" procedural overfitting"; see, e.g., Yarkoni and Westfall, 2017) and trainable parameters (classical overfitting)? How to deal with the fact that experimental studies consist of a number of participants with variable performance, whereas typically only one model instance is evaluated at a time? How to ensure that the reference behavioral data is even representative of the phenomenon of interest, considering finite samples, various biases, and especially the replicability crisis in psychological research (Ioannidis, 2005; Open Science Collaboration, 2015)?

Some of these issues have received some attention in work discussing how to assess the explanatory adequacy of a theory or model. For example, Robinaugh et al. (2021) convincingly argue that the appropriate way in which a model can be compared to a dataset is by having the model generate behaviors similar to those measured in participants, a recommendation we ascribe to here. Yarkoni (2020) claims that the replicability crisis not only may ensue from questionable publication practices and relatively noisy measures across participants. Instead, lack of generalization could well be due to the fact that psychological phenomena are sensitive to factors researchers do not treat as "random effects" as often as they do participants, including factors like stimuli, procedure, and even experimenter. As we will explain in more detail below, one response to this issue precisely will be to stop relying on individual studies, and turn instead to groups of studies (e.g., via meta-analyses; Cristia et al., 2021).

In this paper, we provide additional answers to the above questions and describe a basic conceptual formulation for computational model evaluation focusing on infant behavioral data. As a part of this, we make use of the recently emerged resources for collaborative science in the form of publicly available experimental findings and meta-analyses derived from them (Bergmann et al., 2018; Frank, Bergelson, Bergmann, Cristia, Floccia, Gervain, Hamlin, Hannon, Kline, Levelt, et al.,

\footnotetext{
${ }^{2}$ Another critical component is the ecological plausibility, such as quality and quantity, of the data used for model training. However, this topic is beyond our present scope. See Dupoux (2018) for a discussion.

${ }^{3}$ Any computational model of value should generalize beyond the specifics of its training data by also fitting to previously unseen or yet non-existent data. Overfitting refers to a situation where the model captures the structure of data used for model creation, but the model does not generalize to new samples (see, e.g., Bishop, 2006, or Yarkoni and Westfall, 2017).
} 
2017b). We exemplify our approach using two language capabilities and two alternative computational models for speech pattern discovery. The aim is to steer modeling practices towards more representative and accurate models of infant language learning - models that could gradually get a handle on both the big picture and small details of infant language learning, hence supporting further empirical research with more holistic mechanistic explanations and novel empirical predictions of early language learning. We also highlight some practical and conceptual issues in the behavioral validation of models for which the present work does not provide complete solutions.

\section{Previous work}

A vast number of computational models have been used to study various aspects of early language development - far too many to comprehensively cover them here. A basic modeling approach consists of an unsupervised pattern discovery algorithm that processes spoken language input and learns linguistic patterns from it. The model is usually implemented using a combination of digital signal processing operations, heuristic rules and priors, and/or machine learning algorithms that focus on extracting statistical regularities from the input.

In terms of input, some models operate on linguistic abstractions of speech, such as phonemic, phonetic or orthographic transcripts (e.g., Frank et al., 2010; Goldwater et al., 2009; Nikolaus and Fourtassi, 2021), phonetic or lexical representations derived using pre-trained automatic speech recognition systems (e.g., Fourtassi and Dupoux, 2014; Roy, 2005; Salvi et al., 2012), or by using some simplified representations of acoustic speech, such as formant frequencies of pre-segmented vowels (Coen, 2006; de Boer and Kuhl, 2003). Another set of models operate directly on real continuous speech (e.g., Kamper et al., 2016; Nixon, 2020; Park and Glass, 2008; Räsänen et al., 2015; Schatz et al., 2021; Shain and Elsner, 2020). Besides processing language input only, there are models that use visual concurrent input in addition to spoken language (e.g., Alishahi et al., 2017; Chrupała et al., 2017; Coen, 2006; Harwath et al., 2019; Harwath et al., 2016; Khorrami and Räsänen, 2021; Nikolaus and Fourtassi, 2021; Roy, 2005). Besides passive perception approaches, there are also models that can interact with simulated or real human caregivers (e.g., Howard and Messum, 2011; Rasilo and Räsänen, 2017) and studies using multiple computational agents that can interact with each other using some communicative means (e.g., Kirby, 2001; Moulin-Frier et al., 2015; Oudeyer, 2005; see also Oudeyer et al., 2019, for a recent review).

What is common to all these modeling approaches is that they try to learn some aspects of linguistic patterns or communicative behaviors as part of their operation. The researchers are typically interested in the critical learning components and mechanisms required for successful language learning in the simulations, and sometimes also what type of data is needed for the learning to succeed. In order to evaluate the models, some metrics sensitive to the phenomenon of interest are applied to model after (and sometimes during) its training. As is standard in machine learning, evaluation is typically conducted on held-out test data consisting of samples that were not part of model design and parameter estimation (see also Yarkoni and Westfall, 2017).

The existing evaluation practices of computational models of language development can be roughly categorized into two types (Table 1, top): A) models of human learning that are evaluated against linguistic characterizations of the input data, and B) models of human learning whose performance is compared to behavioral human data from a comparable task or multiple tasks. In addition, there is a third category that we wish to include here, which consists of C) unsupervised machine learning algorithms that are used as a means to harness unlabeled speech data in development of some practical speech technology or natural language processing (NLP) application. On another dimension, modeling studies can also be divided into two distinct groups depending on 
Table 1: An overview of evaluation approaches for computational models of language learning.

\begin{tabular}{|c|c|c|}
\hline Reference point? & Pros & Cons \\
\hline $\begin{array}{l}\text { A: evaluation against linguistic } \\
\text { theory and representations }\end{array}$ & $\begin{array}{l}\text { + easy to interpret } \\
\text { + evaluation data can be annotated post-hoc } \\
\text { + provides learnability proofs } \\
\text { + targets probably correlated with (literate) } \\
\text { adult language representations }\end{array}$ & $\begin{array}{l}\text { - does not necessarily align with infant } \\
\text { language skills } \\
\text { - difficult to account for different } \\
\text { developmental stages in infants } \\
\text { - deals with abstractions that may not always } \\
\text { reflect human processing even for adults }\end{array}$ \\
\hline $\begin{array}{l}\text { B: evaluation against behavioral } \\
\text { or other empirical infant data }\end{array}$ & $\begin{array}{l}\text { + aligns with infant language skills } \\
+ \text { can account for developmental stages } \\
+ \text { captures participant-level variation }\end{array}$ & $\begin{array}{l}\text { - often limited reference data } \\
\text { - noisy reference data (e.g., reproducibility } \\
\text { issues) } \\
\text { - quantitative comparison between models } \\
\text { and reference data non-trivial }\end{array}$ \\
\hline $\begin{array}{l}\text { C: evaluation in terms of end- } \\
\text { use speech applications }\end{array}$ & $\begin{array}{l}\text { + relevant for practical speech technology } \\
+ \text { can inform about capability to capture } \\
\text { different types of speech patterns }\end{array}$ & - nothing ensures alignment with human data \\
\hline Number of capabilities? & Pros & Cons \\
\hline $\begin{array}{l}\text { 1: Evaluation on one } \\
\text { language capability }\end{array}$ & $\begin{array}{l}\text { + easier to conduct } \\
\text { + easy to interpret } \\
+ \text { allows focused research questions \& models }\end{array}$ & $\begin{array}{l}\text { - integration to models of other phenomena } \\
\text { often complex (and rarely done) } \\
\text { - increased risk of overfitting-by-design }\end{array}$ \\
\hline $\begin{array}{l}\text { 2: Evaluation on multiple } \\
\text { language capabilities }\end{array}$ & $\begin{array}{l}\text { + higher representativeness w.r.t. } \\
\text { overall language skills } \\
+ \text { can study interaction of multiple phenomena } \\
+ \text { lower risk of overfitting } \\
+ \text { allows evaluation (and development) of } \\
\text { holistic models }\end{array}$ & $\begin{array}{l}\text { - more tedious to conduct } \\
\text { - more difficult to interpret } \\
\text { - model comparison less straightforward } \\
\text { - models often more complex }\end{array}$ \\
\hline
\end{tabular}

whether the models are evaluated with respect to one or several language capabilities at the same time (Table 1, bottom).

Comparison against linguistic ground truth is likely the most common approach to model evaluation (Category A). In classical phone(me) category learning studies, the computational model is some kind of speech clustering algorithm that tries to assign input speech samples (acoustic observations, or acoustic features) into phonetic or phonemic categories (Coen, 2006; de Boer and Kuhl, 2003; Vallabha et al., 2007). These assignments are then compared against ground-truth phonetic or phonemic identities of the input samples, as defined by manual transcriptions of the data. More recently, a so-called phonemic ABX-test (Schatz et al., 2013; Schatz et al., 2014) has become widely adopted for evaluating models of phonemic learning (e.g., Chorowski et al., 2019; Dunbar et al., 2017; Kamper and van Niekerk, 2021; Nguyen et al., 2020; Schatz et al., 2021). Instead of analyzing clustering purity, the ABX-test analyzes phonemic discriminability of internal representations learned by a model. In studies focusing on speech segmentation, such as phone (Michel et al., 2016; Räsänen, 2014; Scharenborg et al., 2007), syllable (Räsänen et al., 2018), or word segmentation (Shain and Elsner, 2020), the model is typically a system that has a mechanism to identify temporal positions of unit boundaries in time. These boundaries are then compared to unit boundaries in ground-truth phonetic or word-level annotations. A similar process is applied to segmentation models operating on symbolic input, comparing segmented symbol strings to a linguistic reference for the same data (e.g., Brent, 1999; Elsner et al., 2012; Goldwater et al., 2009).

What is common to all these category A studies is that the learning target for the model is based on linguistic abstractions of how spoken language is structured, and could be hence viewed as a measure of linguistic competence (cf., Chomsky, 1965). This is completely justifiable in terms of understanding what the models are learning and what not, enabling learnability proofs for whenever 
linguistic structures a model succeeds to learn in a satisfactory manner. However, what is not so clear is how these measures should be interpreted from an infant language point of view, as infants' language skills are in constant development throughout the infancy (see also Alishahi, 2010, and Section 3.2).

Models evaluated in terms of their fit to experimental laboratory data or observational data form another category of approaches (Category B in Table 1). These models are often evaluated by comparing qualitative patterns in model output and human responses in different experimental conditions (e.g., Frank et al., 2009; Räsänen and Rasilo, 2015; Schatz et al., 2021), or by attempting to mimic changes in task performance as observed in human developmental trajectories (change in the language capability as a function of infant's developmental stage), as defined with respect to some established understanding of the phenomenon in the literature (e.g., Nikolaus and Fourtassi, 2021). Another strategy is to compare model outputs to data from behavioral experimental conditions using standard goodness-of-fit criteria, such as maximum a posteriori (MAP) or maximum-likelihood approaches (ML), Akaike information criterion (AIC), BIC, or some measure of variance explained (e.g., $R^{2}$ or $\eta^{2}$ ), depending on the details of the evaluation setup (e.g., Kachergis et al., 2017; Yurovsky and Frank, 2015). However, these measures are only applicable to parametric models with a tractable likelihood function (see Yarkoni and Westfall, 2017, for a discussion), hence excluding a large number of modeling approaches. Also, while comparison against infant data would be ideal, some of these studies (e.g., Yurovsky and Frank, 2015) have used experimental findings from adults as a basis for model development. Considering the difficulty of conducting experimental research on young babies, the use of adult data may also provide useful insights into the cognitive mechanisms underlying (language) learning in humans. However, as with the linguistic ground truth evaluation (Category A), it is difficult to relate findings from adults to particular stages of infant development.

Unsupervised speech processing algorithms, primarily intended as components in larger speech technology applications, and the related evaluation practices form the third category in our taxonomy (Category $\mathrm{C}$ in Table 1). These algorithms are often evaluated in terms of how they affect the performance of the larger system they are part of, such as word error rates in automatic speech recognition (e.g., Baevski et al., 2020), accuracy of speaker verification systems (Zhang et al., 2021), performance of language models trained on acoustic speech (Kharitonov et al., 2021), or accuracy in audiovisual retrieval tasks (e.g., Harwath et al., 2016). Another alternative is to use so-called diagnostic classifiers to probe the types of information encoded by the learned representations (e.g., performing speaker or phoneme classification using the learned representations as speech features; e.g., Chung and Glass, 2019; Oord et al., 2018), where the aim is to understand the potential of the method for different speech processing use cases. While category $\mathrm{C}$ algorithms are not intended as models of infant learning as such, we wish to point out that the impact that an unsupervised learning module has on an end-system performance may be indicative of the broader relevance of such a mechanism for understanding unsupervised speech pattern acquisition. Hence, we should also consider promising engineering solutions to practical speech processing problems as serious contenders for plausible explanations of language learning from language data, for instance by applying similar model tests to assess fit with respect to human data.

\subsection{Model evaluation on multiple criteria}

The traditional approach has been to model one aspect of language learning at a time, and evaluation has been similarly focused on the language capability in question. However, with the recent emergence of increasingly complex and powerful speech processing and NLP algorithms, there has been increasing interest to understand more broadly what these models are actually learning from 
the data besides how they serve some application-specific needs (see also Belinkov and Glass, 2018, for an overview).

In terms of language models in NLP, Ribeiro et al. (2020) recently proposed CheckList, a wishlist of capabilities that a competent language model should satisfy. To this end, they suggest to subject the model to a number of systematic tests across a range of language capabilities. For instance, the authors suggest to subject any model to verification of functionality in terms of relevant vocabulary and part-of-speech tagging, taxonomy (synonyms, antonyms etc.), robustness against irrelevant input changes, appropriate understanding of relevant named entities, and ability to handle negation, coreference, semantic role labels and logic. In addition, CheckList is an open source tool, and users are invited to contribute to it by including additional tests relevant to a language model (see Ribeiro et al., 2020 for details).

Another branch of research has focused on understanding the similarities and differences between distributional semantics of language models trained on text and lexical semantics in humans. In this context, Lake and Murphy (2021) have argued that we should go beyond comparing human and machine word association scores and synonymity ratings, which has been a standard approach in this area since introduction of Latent Semantic Analysis (Landauer and Dumais, 1997; see, e.g., De Seyne et al., 2021, for a more recent work). Lake and Murphy argue that synonymity and association scores only provide a limited picture of what learned representations of word semantics should capture in order to serve the purposes of human-like communication skills. As an alternative, they propose a list of desiderata for a model of psychological semantics, including possibility to describe or comprehend a scene, linking internal goals and desires to words, understanding requests and instructions, producing novel conceptual combinations, and changing one's beliefs based on linguistic input (Lake and Murphy, 2021).

In terms of acoustic speech-based models, there has been two main branches of research advocating the evaluation of models across several language capabilities. First of these, a series of so-called Zero Resource Speech ("ZeroSpeech") challenges, has driven the development of unsupervised speech learning algorithms through competitions with standardized model training and evaluation protocols (Alishahi et al., 2021; Dunbar et al., 2019; Dunbar et al., 2017; Nguyen et al., 2020; Versteegh et al., 2015). The evaluation batteries in ZeroSpeech challenges started from measuring phonemic selectivity and word segmentation and clustering (Dunbar et al., 2017; Versteegh et al., 2015), and later evolved into tasks that also measure speech production capability and symbolic encoding efficiency of the models (Dunbar et al., 2019). Most recently, the challenge has focused on measuring auditory (Nguyen et al., 2020) and audiovisual learning (Alishahi et al., 2021) of speech patterns in terms of phonemic, lexical, syntactic, and semantic measures.

In parallel to ZeroSpeech, research on so-called visually grounded speech (VGS) models has given rise to an array of metrics to understand what these models are learning. In short, modern VGS models (e.g., Harwath et al., 2019; Harwath et al., 2018) are neural networks that learn statistical correspondences between visual images (or videos; Rouditchenko et al., 2021) and concurrent speech related to the contents of the visual input. Since these models demonstrate emerging understanding of the semantics between auditory speech and the visual world without ever being explicitly taught about the structure of either modality, researchers have become interested on whether the internal representations of these models also show signs of emergent linguistic organization. Studied VGS model capabilities have included, e.g., phonetic, syllabic, and lexical representations and their temporal segmentation, lexical semantics, and, e.g., temporal competition in word activations (Alishahi et al., 2017; Chrupała et al., 2017; Harwath and Glass, 2019; Havard et al., 2019; Khorrami and Räsänen, 2021; Merkx et al., 2019; see also Chrupala, 2021, for a recent review). At the time of writing, the so-far distinct activities in Zerospeech and VGS evaluation have become integrated in a new multimodal extension of the ZeroSpeech challenge (Alishahi et al., 2021). 
The advantage of evaluating across multiple language capabilities is that it gives a much more comprehensive picture of what a model is doing and learning. In fact, the process is necessary for models that explain multiple aspects of language processing at the same time. It also reduces the risk of overfitting the model to (usually limited) data related to a single language capability, and helps to avoid excessive modeling attempts on individual phenomena that cannot be understood in isolation from others. The downside is that measuring and interpreting models from multiple perspectives can be more tedious and complex, both technically and conceptually. Also, the additional measures used to capture language capabilities are typically not the metric the models optimize (e.g., loss functions in neural networks), which makes evaluation-based model improvement less straightforward. Finally, the more there are complementary evaluation measures at play, the more difficult it becomes to rank different models or improvements in one model in cases where gains in some metrics lead to decreased performance in others.

\subsection{Summary of the previous research}

A large proportion of the individual modeling studies and practically all the batteries of tests discussed above focus on representations motivated by linguistic theory and/or measurement with respect to adult-like language competence. While they may represent the ultimate endpoint of an ideal language learner, they cannot capture the developmental stages from birth to the adult-like language competence. And this is a terribly long path to model in one go, especially considering that there may be many developmentally relevant ${ }^{4}$ and informative intermediate stages in the process, and, since language development is not linear, progression of all capabilities from zero to perfect mastery. When modern computational models are pitted against behavioral data from experiments, these data often originate from adult experiments. Only few studies try to match with infant data, in which case the focus is typically on just one language capability at a time, typically based on a single study with a small number of participants. Alternatively, the comparison is largely qualitative in terms of observed outcomes vs. earlier infant reports, which leaves room for interpretation. This is at least partially explained by the difficulty of comparing model responses and infant data on some comparable scale. Finally, the infant data is notoriously noisy and reliability of individual behavioral studies cannot be taken for granted (cf., Oakes, 2017; Open Science Collaboration, 2015). This raises the concern of what empirical data to fit to, and how should one measure the goodness model fit to the given data.

In the next section, we will discuss how and to what extent empirical data on infant language learning could be incorporated to computational modeling practices, what are the advantages and challenges in doing so, and what kind of conclusions can be potentially drawn from such comparisons.

\section{Conceptual framework for model evaluation}

Our aim is to compare computational models against behavioral data from infant language learning experiments in order to evaluate how well the models explain the variability in the human data. Instead of focusing on individual studies and their individual experimental conditions, we concentrate on comparing models against the most robust effects observed in the literature: those supported by systematic meta-analytic findings across a large number of studies. More specifically, we propose an evaluation framework that allows testing of whether a model 1) replicates the systematic findings

\footnotetext{
${ }^{4}$ As an example: If we want to understand language development in 12-month-olds, including to potentially identify risk of atypical development, we need a model of the process that fits to infant data in the same age range- not a model that either is generally adult-like or is not adult-like.
} 
Table 2: An overview of the proposed evaluation guidelines.

\begin{tabular}{l|l}
\hline$\# 1$ & $\begin{array}{l}\text { Robust population-level reference data for computational model evaluation can } \\
\text { be derived from a meta-analysis of behavioral experiments on the language ca- } \\
\text { pability in question. [Section 3.2] }\end{array}$ \\
\hline$\# 2$ & $\begin{array}{l}\text { Meta-analysis can provide reference effect sizes as a function of key moderators } \\
\text { of interest, and, in some cases, null effects of large-scale meta-analyses can be } \\
\text { treated as null targets for the models. [Section 3.2] }\end{array}$ \\
\hline$\# 3$ & $\begin{array}{l}\text { Meta-analyses should be based on up-to-date empirical data when possible. [Sec- } \\
\text { tion 3.2] }\end{array}$ \\
\hline$\# 4$ & $\begin{array}{l}\text { A computational test for a capability should be representative of the capability } \\
\text { in question, aiming to replicate the conditions of behavioral tests behind the } \\
\text { human data (if also satisfying Guideline \#5). [Section 3.3] }\end{array}$ \\
\hline$\# 5$ & $\begin{array}{l}\text { A computational test for a language capability should be flexibly applicable to } \\
\text { various types of models with minimal assumptions on model structure. [Section } \\
3.3]\end{array}$ \\
\hline$\# 6$ & $\begin{array}{l}\text { The amount of model training data can be used as a proxy for the age of children } \\
\text { that are being modeled. [Section 3.3] }\end{array}$ \\
\hline$\# 7$ & $\begin{array}{l}\text { A computational model should reach an effect size comparable or larger to em- } \\
\text { pirically observed human effect sizes in the same task in order to be compatible } \\
\text { with the human data. [Section 3.4] }\end{array}$ \\
\hline$\# 8$ & $\begin{array}{l}\text { Testing a model across multiple language capabilities reduces the risk of over- } \\
\text { fitting to behavioral data, and is needed for the development of more holistic } \\
\text { models of language development. [Section 3.5] }\end{array}$ \\
\hline$\# 9$ & $\begin{array}{l}\text { Leave-one-capability-out (LOCO) evaluation can be applied for principled model } \\
\text { optimization with a reduced risk of overfitting to the phenomena of interest. } \\
\text { [Section 3.5] }\end{array}$ \\
\hline$\# 10$ & $\begin{array}{l}\text { Language capability tests for computational models should be openly docu- } \\
\text { mented and shared to facilitate collaborative science. [Section 3.5] }\end{array}$
\end{tabular}

that exist in the human data, 2) replicates null effects when these are supported by considerable statistical power, and 3) replicates the patterns of effects as a function of key independent variables that can be modified in computational studies, especially infant age (model language experience) in order to account for developmental trajectories. ${ }^{5}$ In addition, our goal is to champion evaluation practices that aim at 4) comparing model and infants across several language capabilities.

Fig. 1 illustrates the conceptual framework for evaluating one chosen language capability with these aims in mind. We will start by formally describing the basic concepts in the framework, and different parts of the process are then described in the following subsections. In Section 4, we demonstrate the process in practice by testing two computational models of speech representation learning on two different language phenomena, namely infant-directed speech (IDS) preference and native/non-native vowel discrimination. As we walk through the steps of our approach, a list of general high-level guidelines will be summarized along the way. Table 2 provides a heads-up summary of these guidelines for orientation.

Since infant learners are facing acoustic speech as the signal, and since acquisition of basic

\footnotetext{
${ }^{5}$ Note that infant developmental trajectories are driven by both maturation and experiences. Ideally, computational models would also include both components, although, as far we know, there is little existing modeling work in this direction.
} 


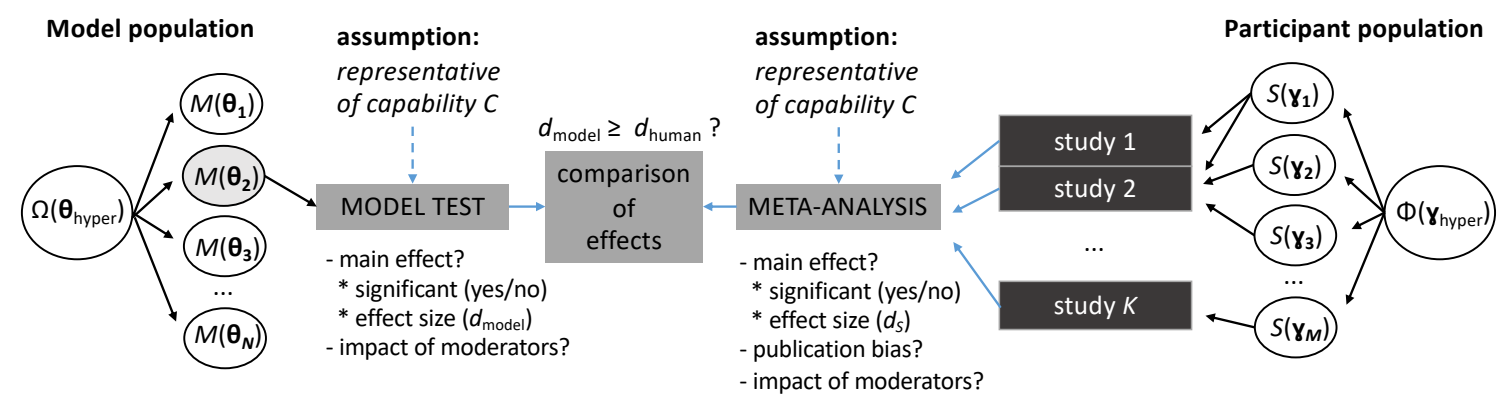

Figure 1: A schematic overview of the proposed evaluation protocol for one language capability. In this example, one particular model instance $M\left(\boldsymbol{\theta}_{2}\right)$ is evaluated against outcomes from a metaanalysis that is based on $K$ empirical studies.

perceptual representations of spoken language is still one of the big questions in modeling research (e.g., Dupoux, 2018; Nguyen et al., 2020), this work focuses purely on models that can also operate on continuous acoustic speech input. However, the evaluation practices we describe should also be applicable to models that use some type of abstract representations of speech, as long as these representations can be systematically derived and substituted for real speech in model training and evaluation. Also note that this work is focused on the special case of evaluating one model instance. The general case of comparing a population of models to a population of humans requires a separate treatment, and is left for future work.

\subsection{Basic definitions}

We define a computational model $M(\boldsymbol{\theta})$ with parameters $\boldsymbol{\theta}$ as a computational implementation of an algorithm, which processes training input $\mathbf{X}_{T} \in[\mathbf{x}(0), \mathbf{x}(1), \ldots, \mathbf{x}(T)], \mathbf{x} \in \mathbb{R}^{d}$, (e.g., speech observations) for time steps $t \in[0,1, \ldots, T]$ up to time $T$ in order to potentially update its parameters due to the input. In addition, we assume that the model can be probed for two types of variables for each input $\mathbf{x}(t)$ (top in Fig. 3): internal representations $\mathbf{z}(t)=f\left(\mathbf{x}(t), \mathbf{x}(t-1), \ldots \mid \boldsymbol{\theta}_{t}\right) \in \mathbb{R}^{h}$ and attentional preference scores $a(t)=f\left(\mathbf{x}(t), \mathbf{x}(t-1), \ldots \mid \boldsymbol{\theta}_{t}\right) \in[-\infty, \infty]$. The internal representations correspond to $h$-dimensional vectors of activations caused by sensory input and other potential internal states of the model up to that point in time. The attentional preference score stands for model's internal rating of how strong of an attentional attractor is the stimulus (compared to other potential stimuli separated in time). We may further divide $\boldsymbol{\theta}$ into two distinct sets: $\boldsymbol{\theta}_{\text {adapt }}$ that can change due to input data and hyperparameters $\boldsymbol{\theta}_{\text {prior }}$ that are set before model training.

In order to consider variation across a number of similar models with different initial parameters and/or hyperparameters, we say that a model comes from a population of models $M(\boldsymbol{\theta}) \in \Omega\left(\boldsymbol{\theta}_{\text {hyper }}\right)$ of the same type, where initial values of $\boldsymbol{\theta}_{\text {adapt }}$ and the fixed values of $\boldsymbol{\theta}_{\text {prior }}$ of $M$ are sampled from a hyperprior distribution $\boldsymbol{\theta}_{\text {hyper }}$ shared by all members of the population (Fig. 2).

In analogy, we can consider each human participant $S(\gamma)$ of a scientific experiment to be sampled from a population $\phi\left(\gamma_{\text {hyper }}\right)$. We can furthermore assume that each human participant can be probed for an overt behavioral response $\mathbf{a}(t) \in \mathbb{R}^{k}$ for stimulus $\mathbf{x}(t)$. A response that is dependent on intermediate encoding $(\mathrm{s}) \mathbf{z}(t)=f_{z}\left(\mathbf{x}(t), \mathbf{x}(t-1), \ldots \mid \boldsymbol{\gamma}_{t}\right) \in \mathbb{R}^{f}$ similarly to our models (bottom in Fig. 3). While human internal states $\mathbf{z}(t)$ are not directly observable, we can use some instrumentation (e.g., fMRI or EEG) to measure some vector $\mathbf{y}(t)=f_{y}(\mathbf{z}(t))=f_{y}\left(f_{z}(\mathbf{x}(t))\right) \in \mathbb{R}^{n}$ from the 
participant's brain for each $\mathbf{x}(t)$, reflecting the properties of encoding $\mathbf{z}(t)$.

Finally, we assume that the time-dependent model responses $a(t)$ and $\mathbf{z}(t)$ and human responses $\mathbf{a}(t)$ and $\mathbf{y}(t)$, when measured for $i$ :th trial of an arbitrary experiment, can be converted to some scalar measure $\alpha_{i}$ that stands for the model/participant response for the given trial. To give a concrete example, an infant response $\mathbf{a}_{i}(t)$ during trial $i$ could be the full array of motor activity, including torso, head, and eye orientation, whereas $\alpha_{i}$ would be the resulting measured and recorded looking time at a target stimulus from that trial, given the particular measurement setup. For an event-related potential (ERP) brain study, an analogical process would be the averaging of an electrode response $y_{i}(t)$ for trial $i$ across a pre-defined time window to obtain the $\alpha_{i}$ (for computational model examples, see Section 4).
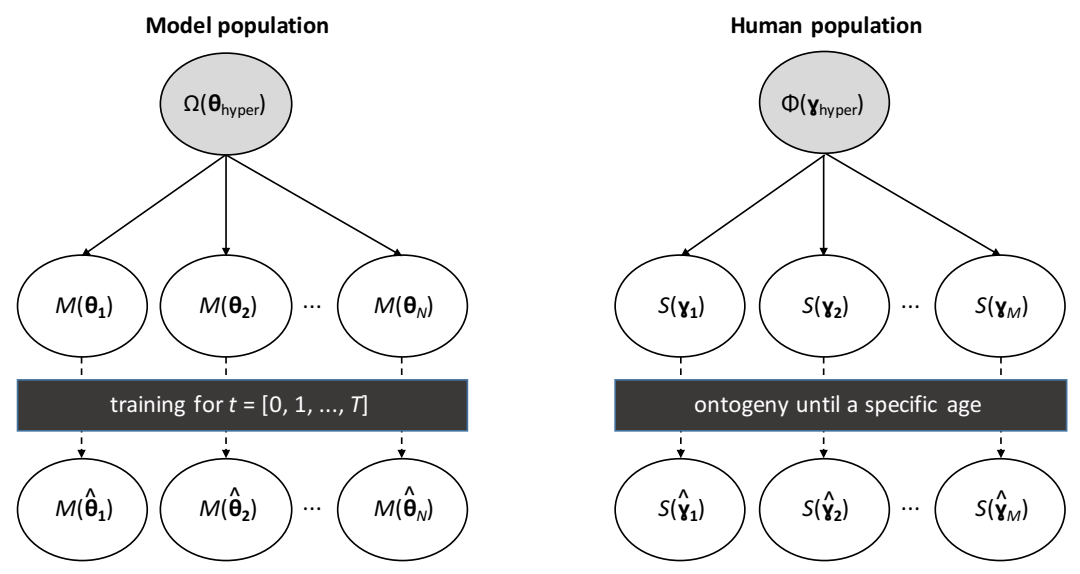

Figure 2: The parallel between model instances and human participants, and how model parameters become defined. Hyperpriors $\boldsymbol{\theta}_{\text {hyper }}$ (models) and $\boldsymbol{\gamma}_{\text {hyper }}$ (humans) define variation across individuals before training or development with particular life experiences has taken place. By training models with a certain set of data, we aim to simulate a certain developmental stage of human participants.

In order to know if a model fits to human data in terms of a chosen language capability, we need to study whether the responses of a model are comparable to those of human participants. Considering a typical infant experiment, one collects responses $\alpha_{A}=\alpha_{A, 1}, \alpha_{A, 2}, \ldots$ for trials in condition $\mathrm{A}$ compared to responses $\alpha_{B}=\alpha_{B, 1}, \alpha_{B, 2}, \ldots$ for trials in condition $\mathrm{B}$, such as measuring looking time towards novel (A) compared to familiar (B) words, and where the difference between the conditions $\mathrm{A}$ and $\mathrm{B}$ is only related to the capability of interest. We furthermore assume each $\alpha_{A} \in \alpha_{A}$ and $\alpha_{B} \in \alpha_{B}$ to be independent and identically distributed (i.i.d) for convenience of notation. One is then interested in i) whether the responses in the two conditions are statistically different, and if so, ii) how big this effect is, i.e., what is the effect size. By simplifying the math to its core (ignoring the sign, assuming equal number of samples for A and B), calculation of the effect size is essentially proportional to the difference in responses to the conditions, normalized by the standard deviation (or variance) of the data:

$$
d \sim \frac{\left|\mu_{\alpha_{A}}-\mu_{\alpha_{B}}\right|}{\sigma_{\alpha}}
$$

where $\mu$ are the condition means and $\sigma$ is the standard deviation of the measurements $\alpha$. In some designs (e.g., within-participant experiments), the effect size is derived from difference scores 

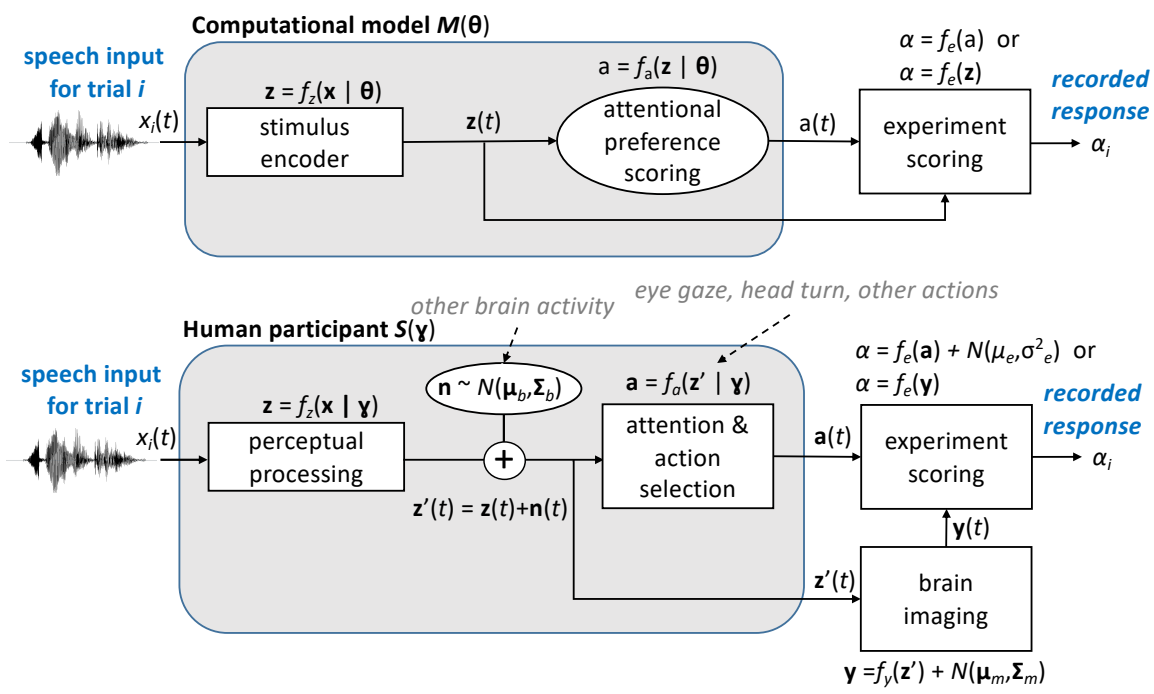

Figure 3: A simplified schematic view on measuring a model vs. measuring a human participant. With models, direct access to model internal states $\mathbf{z}(t)$ and attentional preference scores $a(t)$ is available without additional measurement uncertainty. These are then mapped to recorded responses $\alpha_{i}$ for each test trial $i$ using some test-specific function $f_{e}()$. With human participants, recorded responses $\alpha_{i}$ are assumed to be a function of input stimuli, but also depend on other factors, such as other ongoing brain/cognitive activity (modeled as $\sim N\left(\boldsymbol{\mu}_{b}, \boldsymbol{\Sigma}_{b}\right)$ ), measurement noise in the particular behavioral setup $\left(\sim N\left(\mu_{e}, \sigma_{e}^{2}\right)\right.$, or noise in the brain imaging approach $\left(\sim N\left(\boldsymbol{\mu}_{m}, \boldsymbol{\Sigma}_{m}\right)\right)$. Variables for time $(t)$ omitted from the function definitions for the sake of visual clarity.

$\alpha_{\text {diff }}=\alpha_{\mathrm{A}}-\alpha_{\mathrm{B}}$ of pre-defined trial pairs with

$$
d \sim \frac{\left|\mu_{\alpha_{\text {diff }}}\right|}{\sigma_{\alpha}},
$$

In other words, Eqs. 1 and 2 measure how different is the average reaction between the two experimental conditions, normalized by variability across the measurements.

Since effect-size is a scale-free metric, it is in theory suitable for comparing models to humans in basically any behavioral experiment. In order to compare human and machine data, we will simply need an estimate of $d_{\text {human }}$ for human participants and $d_{\text {model }}$ for our model for the language capability in question. Naturally, one will also need a model that can "participate" in an experiment that taps the capability in question.

\subsection{Human reference from meta-analysis}

In order to have human reference effect sizes $d_{\text {human }}$ for a particular language capability $C$, we need to look into empirical data on infant language processing. Even though there is substantial value in trying to computationally replicate individual studies and studying how various experimental manipulations affect human responses, the replication issues in experimental research (Open Science Collaboration, 2015) clearly demonstrate that the results from individual conditions of individual studies do not necessarily generalize to the population at large. This raises the question of what type of behavioral reference can be used to guide model development and evaluation. 
In this context, there are two ways in which the experimental community can provide results that are most useful and reliable. First, large-scale collaborations where multiple labs run the same or similar studies provide unbiased and generalizable results. The ManyBabies consortium was set up precisely with this aim (Frank, Bergelson, Bergmann, Cristia, Floccia, Gervain, Hamlin, Hannon, Kline, Levelt, et al., 2017a), and has already started 5 such collaborative projects. The first of these projects has already been published, and will be used in Experiment \#1 (see Section 4).

The second way in which infant data may be retrieved is via meta-analysis, an increasingly common approach in early language development (Bergmann et al., 2018; Lewis et al., 2016). The goal of meta-analysis is to include experiments across different samples, research methods, labs, languages, or other factors that may add variability to the data, together offering a higher statistical power, greater coverage, and a more realistic characterisation of the underlying phenomenon than that of an individual study. Meta-analyses can be used to derive the current best estimate of the effect size associated with the underlying phenomenon (via the weighted mean across all included studies); and to estimate the extent to which potential moderators actually explain differences in effect sizes across different experiments. The multivariate meta-analysis model is particularly convenient in early language development research, where apart from effect size distribution and mean effect size, researchers are also interested in developmental trajectory analyses. By using age as the independent variable (or moderator), the multivariate meta-analysis model can reveal changes in effect size as a function of infants' age (see, e.g., Lewis et al., 2016), hence providing developmental trajectory targets for models to replicate.

In the context of infant language, a collaborative resource called MetaLab (Bergmann et al., 2018; Lewis et al., 2016) ${ }^{6}$ is a practical solution to meta-analytic data access, as it integrates data from different studies and labs to an open database. At the time of writing, the database houses studies on 24 categories of early language studies (here referred to as different language capabilities) from hundreds of published papers and unpublished reports, includes links to meta-analyses published on the data, and the database is updated in terms of new meta-analyses and data contributions from the research community. All this makes MetaLab a very attractive source of reference data for model evaluation, as it has both up-to-date data and, for most of the capabilities, reports describing the meta-analytic practices to analyze these data for the developmental phenomena of interest, which allows computational modelers that are not experts on infant experimentation to adopt the meta-regression specification decided upon by the meta-analysis authors. In the age of cumulative science, such "community augmented meta-analyses" are likely the best approach to evaluating a theory's explanatory adequacy (i.e., its fit to extant data; Cristia et al., 2021), including because they minimize selection bias, they invite checks for scope (i.e., whether the whole space of design and stimuli is appropriately represented), and they use statistical techniques to actually account for potential effect variation. We will be drawing from MetaLab for our Experiment \#2 (see Section 4).

Regardless of whether data come from a large-scale experiment or a meta-analysis, we propose a specific sequence of steps for deriving human reference data to test a computational model. Once the data have been obtained, one identifies the key moderators of interest that both are available in the literature and can be manipulated in model testing (e.g., infant age or native language). Note that some additional moderators explain heterogeneity in the infant data but are irrelevant to computational tests (e.g., specifics of the experimental methodology to probe infants' knowledge, such as eye-tracking vs. head-turn preference), and could be included as as covariates (to soak up variance in infant data, but not to compare their effects with the analogous effects in models). Finally, one extracts the relevant effect sizes $d_{\text {human }}$ and their confidence intervals, as calculated in the selected meta-analysis. In our experiments, we will start by assessing whether there is a main

\footnotetext{
${ }^{6}$ http://metalab.stanford.edu/
} 
effect, and then whether this is affected by a computationally-relevant moderator, age. To derive a developmental trajectory (effect size as a function of infants' age, or amount of exposure for the model), we also recommend following the approach taken in the original meta-analysis study, where in some cases, the trajectory is extracted using the multivariate model (which will thus include covariates), while some others choose to model the effect size as a function of age only, all this assuming that the slope estimate for the age is significant. As a result, the process produces effect size targets for the model to reach as a function of the key moderators (see Fig. 4 for an illustration). Null effects are ambiguous: Although theoretically they can still act as a meaningful target for a model (i.e., the model should also have null effect for this capability), they may also signal noisy infant data (see Section 3.4), which is an issue particularly for moderators (for which power is always lower than for main effects; see also Lewis et al., 2016; and Steps 9 and 0 in Cristia et al., 2021). In the absence of better knowledge, we will treat null effects as null targets for models as well, especially if the null finding is supported by substantial statistical power.
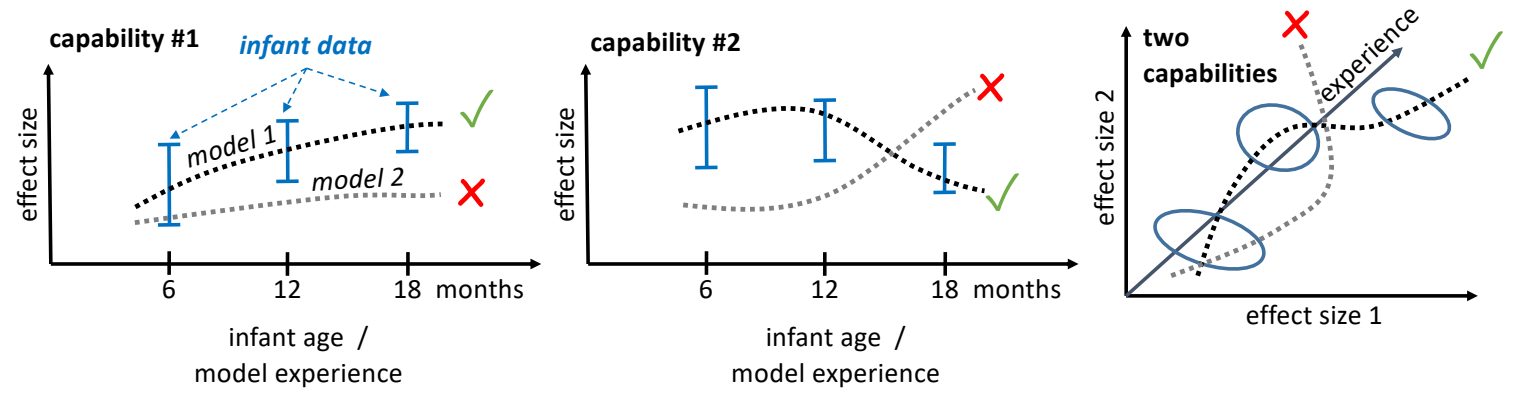

Figure 4: Illustration of an ideal evaluation setup, where we would be able to accurately compare human and model effect sizes on a similar scale. In the first two panels, the blue error bars show infant effect sizes (as their 95\% confidence intervals) derived from meta-analyses for 6, 12, and 18 month-old infants for two imaginary language capabilities. In addition, effect sizes measured for two imaginary models, tested as a function of the amount of model training data, are also shown for each capability. The model with the dark-dotted line fits to the data in both cases, while the one denoted with lighter grey dots does not. The right panel visualizes how the confidence intervals of effect size estimates of two capabilities, when considered concurrently, form hyperspheric behavioral "targets" for the models in a 3-D space - a principle that generalizes to any arbitrary number of concurrently evaluated capabilities.

From a practical perspective, the datasets and existing meta-analyses available through MetaLab already provide a large array of developmental trajectories and milestones across a range of language capabilities. Hence, one practical goal for the modeling community would be to implement a battery of computational tests aligned with those analyses, thereby enabling a holistic evaluation of developed models.

Guideline \#1: For the most robust behavioral reference, models can be compared to effects confirmed in meta-analyses on the given language capabilities instead of fitting them to data from individual studies.

Guideline \#2: Meta-analysis can provide reference effect sizes as a function of key moderators of interest, and, in some cases, null effects of large-scale meta-analyses can be treated as null targets for the models.

Guideline \#3: Meta-analyses should be based on up-to-date empirical data when possible (where MetaLab is currently a practical source for these). 


\subsection{Designing computational tests for language capabilities}

In order to compare a computational model against the effect size from meta-analysis on a language capability, we need to device a computational test for the model that translates measurable model behavior to effect sizes similarly to human data. In addition, the test should also reveal effect sizes as a function of the key moderators of interest, for which reference data points were obtained from the human meta-analysis step.

More formally, given a model $M(\boldsymbol{\theta})$ and a computational test for capability $C$, the test should produce a mean estimate of $d_{\text {model }}$ together with estimates of $d_{\text {model }}$ as a function of chosen moderators $\beta \in B$ of interest. In order to derive the $d_{\text {model }}$, the model is subjected to a set of test trials $v_{i} \in$ $V, i=\{1,2, \ldots, N\}$, each minimally consisting of speech input $\mathbf{x}_{i}(t)=\left\{\mathbf{x}_{i}(1), \mathbf{x}_{i}(2), \ldots, \mathbf{x}_{i}\left(T_{i}\right)\right\}$ of duration $T_{i}$. For each $\mathbf{x}_{i}(t)$ in $v_{i}$, temporal attentional preference scores $a_{i}(t)=\left\{a_{i}(1), a_{i}(2), \ldots, a_{i}\left(T_{i}\right)\right\}$ and/or latent encoded representations $\mathbf{z}_{i}(t)=\left\{\mathbf{z}_{i}(1), \mathbf{z}_{i}(2), \ldots, \mathbf{z}_{i}\left(T_{i}\right)\right\}$ are recorded from the model. In addition, each trial is associated with a list of potential moderator values $\boldsymbol{\beta}_{i}=\left\{\beta_{i, 1}, \beta_{i, 2}, \ldots\right\}$ (e.g., vowel contrast type and natural vs. synthetic stimulus in discrimination studies) and with a function $f_{e}()$ that maps model responses $a_{i}(t)$ or $\mathbf{z}_{i}(t)$ to trial response scores $\alpha_{i}$. Task of the computational test designer is then to i) design and create the stimuli for each $v_{i}$, ii) define the algorithmic instructions $f_{e}()$ to calculate trial-specific response scores from model outputs, iii) and derive the corresponding effect size(s) $d$ from the final set of $\alpha_{i}, i=\{1,2, \ldots, N\}$, and for the moderators $\beta$ of interest (see Fig. 3 for illustration). In general, the exact procedure of deriving the condition-specific responses and resulting effect sizes for the model depends on the capability and test design in question. Some examples of how to derive the responses and calculate effect sizes for typical speech stimulus testing scenarios are given in Appendix A. In addition, two practical examples will be provided in the experiments of Section 4.

As for designing the test structure itself, we rely on two core principles: test representativeness and flexibility. First, we assume that the effect size and moderator interactions from the human meta-analysis are representative of the given language capability in question. By representativeness we mean that the meta-analysis is conducted properly, focuses on the phenomenon of interest (without including irrelevant studies, and fully sampling the potential space of possibilities; see Cristia et al., 2021 for discussion), and is based on up-to-date empirical data, hence reflecting the best possible reference available at a given time. Second, we specify that the computational test should be (equally) representative of the capability in question. What then corresponds to a representative computational test of the capability being studied? The concept of representativeness is naturally an idealization, and a practical test will consist of design choices that will impact the outcomes of the test. As in meta-analyses, the computational test should involve several different conditions, thus ensuring appropriate coverage of capability-relevant variability in the stimuli. These sources of variability include, e.g., the use of multiple different contrasts in a vowel discrimination task or counterbalanced conditions for artificial language learning tasks. In addition, extralinguistic variability present in real speech and across different behavioral experiments should also be considered, such as using diverse speakers and speaking styles, or using both natural and synthetic speech stimuli. A practical starting point is to pursue replication of the experimental setups utilized in behavioural studies $^{7}$, potentially adapted to the special characteristics and opportunities of computational testing that allow much more accurate and extensive tests (e.g., the total number of trials is not limited similarly to human subjects). Ultimately, representativeness seeks to ensure that the capability analysis does not depend on the particulars of one test scenario, but rather on an aggregate of multiple scenarios.

\footnotetext{
${ }^{7}$ In fact, if several experimental paradigms are replicated as computational tests, the experimental paradigm can be used as a moderator in the human and model effect size analysis, enabling probing of finer setup-dependent effects.
} 
The second key property, flexibility, aims at representative tests that are directly applicable to a wide range of computational models without imposing strong assumptions regarding the models' operating characteristics. We have already defined that each model should be able to process speech inputs $\mathbf{x}_{i}(t)$ to produce responses $a_{i}(t)$ and $\mathbf{z}_{i}(t)$, and the test should rely on those without additional requirements for model inputs and outputs (with the exception of multimodal models, which are not discussed here). Beyond those, many models have constraints on minimum or maximum duration of input signals that can be processed at a time. The model performance may also be affected by "boundary effects" close to onsets or offsets of input signals (e.g., due to recurrent processing or temporal windowing). Hence, very short (some hundreds of milliseconds) or very long (tens of seconds) speech inputs should be avoided unless necessary. ${ }^{8}$ Also, if a capability can be tested without the use of a separate familiarization stage, the test becomes computationally more straightforward to operate. However, some capabilities (e.g., those based primarily on artificial language experiments) will require some type of model learning to take place during the test if a model is to succeed in the task.

While it is impossible to ensure perfect test compatibility with all imaginable models, experience has shown that well-designed standardized tests and metrics for machine learning challenges can be applicable to a range of modeling approaches (e.g., Dunbar et al., 2017; Nguyen et al., 2020). Overall, the tests should aim at being agnostic to how models operate, which implies assessing the capability independently of the assumptions made by models (e.g., models assuming discrete linguistic units should be evaluated in the same manner as models without the discreteness assumption). Our guideline for flexibility invites us to design robust and simple tests.

Note that a computational test cannot manipulate simulated "age" of a model that is being tested, as the learning in the model takes place using a completely independent set of training data. In order to estimate developmental trajectory of a model, the model can be trained with increasing amounts of training data and then tested at predetermined checkpoints. Ideally, these checkpoints would be somehow grounded to particular ages or developmental stages of simulated infants, although there are currently few existing resources that would support accurate simulation of spoken language experiences of individual children across several months of development ${ }^{9}$. In our experiments, we demonstrate developmental trajectory analysis by simply comparing untrained models to those trained with 100 hours of English read speech.

In summary, our guidelines for computational test design are as follows:

Guideline \#4: A computational test for a capability should be representative of the capability in question, aiming to replicate the conditions of behavioral tests behind the human data (if also satisfying Guideline \#5).

Guideline \#5: The computational test should be flexibly applicable to various types of models with minimal assumptions on model structure (besides the standardized speech inputs and attentional preference and latent representation outputs).

Guideline \#6: The amount of training data can be used as a proxy for model "age". This training step is conceptually separated from a computational test for capability $C$ in the same way that development of a child is conceptually separate from any behavioral test administered to the child.

\footnotetext{
${ }^{8}$ Signal duration is different from durations of the stimuli, but one signal should ideally include leading and trailing silence and may consist of several subsequent stimuli.

${ }^{9}$ Daylong child-centered recordings are a potential development towards this direction, but currently come with many challenges related to signal noise and sampling density with respect to individual children (see, e.g., Räsänen et al., 2021, for a discussion).
} 


\subsection{Comparing model effects to human effects}

The last step of evaluating a model on a capability $C$ is to compare the human effect size $d_{\text {human }}$ (Section 3.2) to that of $d_{\text {model }}$ obtained from a model (Section 3.3). If only one model instance $M(\boldsymbol{\theta})$ is being evaluated, there are two potential questions to ask: 1) does the model reflect the average performance of humans, or 2) whether this model is a likely member of the human population in terms of its language capability/capabilities. Unfortunately, meta-analyses based on study-level aggregates do not provide a systematic means to estimate variance of the effect across the participant population (see General Discussion). Instead, meta-analysis provides an estimate of the mean effect size and its error bounds. Hence, we can only try to answer the former question, i.e., check whether the effect size of the model is compatible with the mean population performance, but not whether the model is a plausible model of any individual learner.

In principle, given our assumption of computational test representativeness, one could just subject a model to the test, record the responses $\alpha$, calculate $d_{\text {model }}$, and then compare it to the human result $d_{\text {human }}$ in terms of effect magnitude after checking for a null-effect (see Fig. 4 for illustration). However, the problem is that the effect size estimates for a model are not directly comparable to those of human participants. While one has a completely noise-free access to everything taking place inside a model and to its outputs, measurement of human internal processing can only be achieved by indirect means (Fig. 3). When we measure human responses to different stimuli, we assume that the response is causally related to the input. However, this response is not only driven by the input, but is also affected by everything else going on in the brain and in the current embodied and contextual situation. For instance, the role of task-irrelevant factors is considerable in young babies, adding noise to the process (Peterson, 2016, pp. 3-4). In addition, the available data for any individual behavioral experiment or brain imaging study is not a perfect image of participant behavior in the experiment. In contrast, the data consist of records that are a result of some type of (non-linear) measurement process - a process that can have multiple sources of uncertainty and measurement noise depending on the utilized paradigm. How does this then impact the comparison between models and humans?

As defined in Section 3.1, magnitude of the effect size of an experiment with conditions A and $\mathrm{B}$ is proportional to

$$
d \sim \frac{\left|\mu_{\alpha_{A}}-\mu_{\alpha_{B}}\right|}{\sigma_{\alpha}},
$$

where $\mu$ is the mean and $\sigma$ the pooled standard deviation across the measurements $\alpha$. As illustrated in Fig. 3, any internal state $\mathbf{z}^{\prime}$ in the human cognitive system will be affected by encoding of the stimulus $f_{\mathrm{z}}(\mathbf{x})$ plus noise of stimulus-independent processing, which we can assume to be normally distributed $N\left(\boldsymbol{\mu}_{\mathrm{b}}, \boldsymbol{\Sigma}_{\mathrm{b}}\right)$ with generally unknown mean and covariance (cf., central limit theorem):

$$
\mathbf{z}^{\prime}=f_{\mathrm{z}}(\mathbf{x})+N\left(\boldsymbol{\mu}_{\mathrm{b}}, \mathbf{\Sigma}_{\mathrm{b}}\right) .
$$

In the case of a behavioral experiment, this latent signal gets then transformed into externally measurable motor action a (e.g., measurable attentional orientation) through some unknown function $f_{\mathrm{a}}\left(\mathbf{z}^{\prime}\right)$. This is followed by the experiment-dependent practice $f_{\mathrm{e}}()$ for measuring and recording a response $\alpha=f_{\mathrm{e}}(\mathbf{a})$ for the given trial. In this step, the instrumentation and recording practices of the setup again introduce some extra noise to the measurements (e.g., eye-tracking imprecision and pre-defined hard thresholds for determining a response type):

$$
\alpha=f_{\mathrm{e}}\left(f_{\mathrm{a}}\left(\mathbf{z}^{\prime}\right)\right)+N\left(\mu_{\mathrm{e}}, \sigma_{\mathrm{e}}^{2}\right)=f_{\mathrm{e}}\left(f_{\mathrm{a}}\left(f_{\mathrm{z}}(\mathbf{x})+N\left(\boldsymbol{\mu}_{b}, \boldsymbol{\Sigma}_{\mathrm{b}}\right)\right)\right)+N\left(\mu_{\mathrm{e}}, \sigma_{\mathrm{e}}^{2}\right) .
$$


In the case of brain imaging, we also have a situation where an attempt to measure $\mathbf{z}^{\prime}$ is followed by a potentially non-linear measurement function $f_{\mathrm{y}}\left(\mathbf{z}^{\prime}\right)$ and associated measurement noise $N\left(\boldsymbol{\mu}_{\mathrm{m}}, \boldsymbol{\Sigma}_{\mathrm{m}}\right)$, i.e.,

$$
\mathbf{y}=f_{\mathrm{y}}\left(\mathbf{z}^{\prime}\right)+N\left(\boldsymbol{\mu}_{\mathrm{m}}, \boldsymbol{\Sigma}_{\mathrm{m}}\right)=f_{\mathrm{y}}\left(f_{\mathrm{z}}(\mathbf{x})+N\left(\boldsymbol{\mu}_{\mathrm{b}}, \boldsymbol{\Sigma}_{\mathrm{b}}\right)\right)+N\left(\boldsymbol{\mu}_{\mathrm{m}}, \boldsymbol{\Sigma}_{\mathrm{m}}\right),
$$

which is then transformed with some $f_{\mathrm{e}}()$ to a recorded response $\alpha$ for statistical analysis.

For convenience, let us now define the variance component in the recorded responses that only depends on the stimuli and their encoding as

$$
\sigma_{\text {stimuli }}^{2}=\operatorname{Var}\left\{f_{\mathrm{a}}\left(f_{\mathrm{z}}(\mathbf{x})\right)\right\} .
$$

Now, if we assume all the noise sources to be independent of input stimuli and each other, and even when we don't generally know parameters of the noise distributions or forms of the involved functions, we can still write:

$$
\sigma_{\text {res }}^{2}=\operatorname{Var}\left\{f_{\mathrm{a}}\left(f_{\mathrm{z}}(\mathbf{x})+N\left(\boldsymbol{\mu}_{\mathrm{b}}, \boldsymbol{\Sigma}_{\mathrm{b}}^{2}\right)\right)\right\}-\sigma_{\text {stimuli }}^{2} \geq 0 .
$$

where $\sigma_{\text {res }}^{2}$ now stands for the residual variance between total data variance for processing the stimuli $\mathbf{x}$ into actions $\mathbf{a}$, when other "noise" in the cognitive system is taken into account, and variance solely dependent on stimuli. In addition, for measurement noise we know that

$$
\sigma_{\mathrm{e}}^{2}=\operatorname{Var}\left\{N\left(\mu_{\mathrm{e}}, \sigma_{\mathrm{e}}^{2}\right)\right\} \geq 0 .
$$

With the help of these, we can rewrite the denominator of Eq. (3) as

$$
\sigma_{\alpha, \text { human }}^{2}=\sigma_{\text {stimuli }}^{2}+\sigma_{e}^{2}+\sigma_{\text {res }}^{2} \geq \sigma_{\text {stimuli }}^{2}
$$

whereas for the noise-free computational models, we have

$$
\sigma_{\alpha, \text { model }}^{2}=\operatorname{Var}\left\{f_{\mathrm{a}}\left(f_{\mathrm{z}}(\mathbf{x})\right)\right\}=\sigma_{\text {stimuli }}^{2}
$$

What Eq. (10) means in practice is that the variance of the recorded human responses to experimental stimuli must be equal or larger than the variance that would be solely attributable to the processing of stimulus only. Since the noise distributions are assumed to be independent of the stimuli, they do not affect numerator of Eq. (1). As a result, the observed effect size decreases, and this occurs independently of experimental design (e.g., within- vs. across-participants) and of whether data are aggregated or stem from a single study. In contrast, computational models do not share the same noise terms with human data. Therefore, the effect sizes measured for human participants tend to underestimate the magnitude of effect compared to a computational model, at least as long as 1) the model does not have dedicated noise mechanisms or concurrent task-irrelevant processing mechanisms that would introduce additional processing noise to the model's behavior, and 2) the test setup does not simulate (or introduce) measurement noise comparable to behavioral or brain imaging experiments.

A consequence of the above is that a model reaching $d$ equal or larger to human $d$ on capability $C$ can still be a valid model for the phenomenon (see Fig. 5) (under the assumption of test representativeness discussed in Section 3.3). In contrast, a model reaching significantly lower $d$ compared to human data is incompatible with the human data. Also note that the upper bound of an acceptable model effect size is difficult to estimate, as the amount of noise in human data is generally unknown and varies from one experiment (and paradigm) to another. 

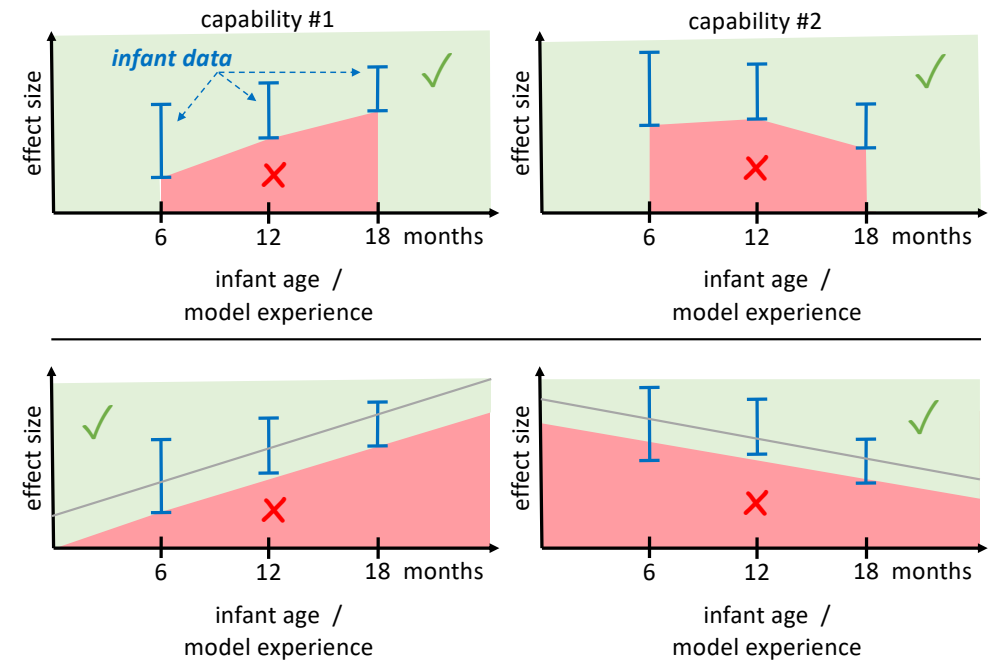

Figure 5: Illustration of a more practical evaluation setup for two language capabilities on the left and right, where direct comparison of model effect sizes is problematic with the human data being inherently more noisy. The blue error bars illustrate $95 \%$ confidence intervals on the human effect sizes acquired from meta-analysis for three infant ages. Top: A stringent scenario, where we expect the model to achieve larger-than-human effect sizes for the quantified age range, but we make no assumptions outside this range (not practical). Bottom: a scenario where a least-squares fit to age interaction in the effect size is used to determine a lower bound for model effect size as a function of age (more practical; needs to be replaced with a polynomial fit for non-linear developmental trajectories).

The direction of effect size change as a function of a given moderator (i.e., the sign of the beta, in a meta-regression) is not similarly affected, if one assumes that the noise terms are independent of the moderators. Thus, whereas the intercept of a meta-regression only provides a lower bound of what a model needs to match, betas provide additional constraints for the model to satisfy. The assumption that noise is independent from the moderators, however, is not true across the board; saliently, it is not true for participant age, where the entire cognitive system undergoes many changes with time (which often result in decreased noise) and also because different experimental paradigms may be used with children of different ages. Additionally, estimators in a meta-regression may be affected by the moderators included in it; for instance, the effect of age may be affected by whether changes according to the use of different experimental paradigm are statistically accounted for. We believe it will be challenging for modelers to make decisions regarding which moderators should be included, and thus it would be ideal that meta-analysts make such decisions. Once such decisions are made to best describe infant data, we recommend that comparisons focus on relative change (rather than absolute magnitudes), where for a significant increase in infant effect size due to moderator manipulation (e.g, increasing infant age) we would expect a compatible model to have a robust increase in effect size as well, and vice versa.

Finally, also note that when testing only one model with a fixed computational test, the resulting effect-size will be, by default, a deterministic point estimate. In contrast, the human effect size from the meta-analysis will have an estimate of the effect mean together with its confidence interval.As far as we know, there is no well-defined statistical procedure to obtain a binary decision (at a certain 
significance level) on whether the model effect size is equal or larger than that of the human one in this situation. Instead, one can simply numerically check whether the model is above the low end of the human confidence interval (e.g., model effect being at the lower bound of the human $95 \%$ interval would have $p<0.025$ of being smaller than that of infants). If the number of trials in the test is large (in the thousands, which we recommend), the effect size should be robust.

Guideline \#7: A computational model or a pool of models should reach an effect size comparable or larger to empirically observed human effect sizes in the same task in order to be compatible with the human data.

\subsection{Testing models across several language capabilities}

One of the grand aims of computational modeling of early language development is to discover models capable of replicating infant language learning across the broad spectrum of language phenomena. By developing models that attempt to explain multiple aspects of language learning as a function of developmental stage and with a finite set of integrated processing mechanisms, we can gradually approach the unified theories of language learning needed to understand the interactions and dependencies between sensorimotor (language) experience, learning mechanisms, and internally represented and externally observable language learning outcomes at individual and population levels (see also Karcheris et al., 2021).

From a model evaluation perspective, this entails combining two approaches: (1) evaluating against infant data and (2) evaluating against multiple linguistic capabilities simultaneously (see Table 1). In this context, a holistic model is expected to exhibit performance compatible with human data across a set of linguistic skills and across the developmental timeline (Figs. 4 and 6).

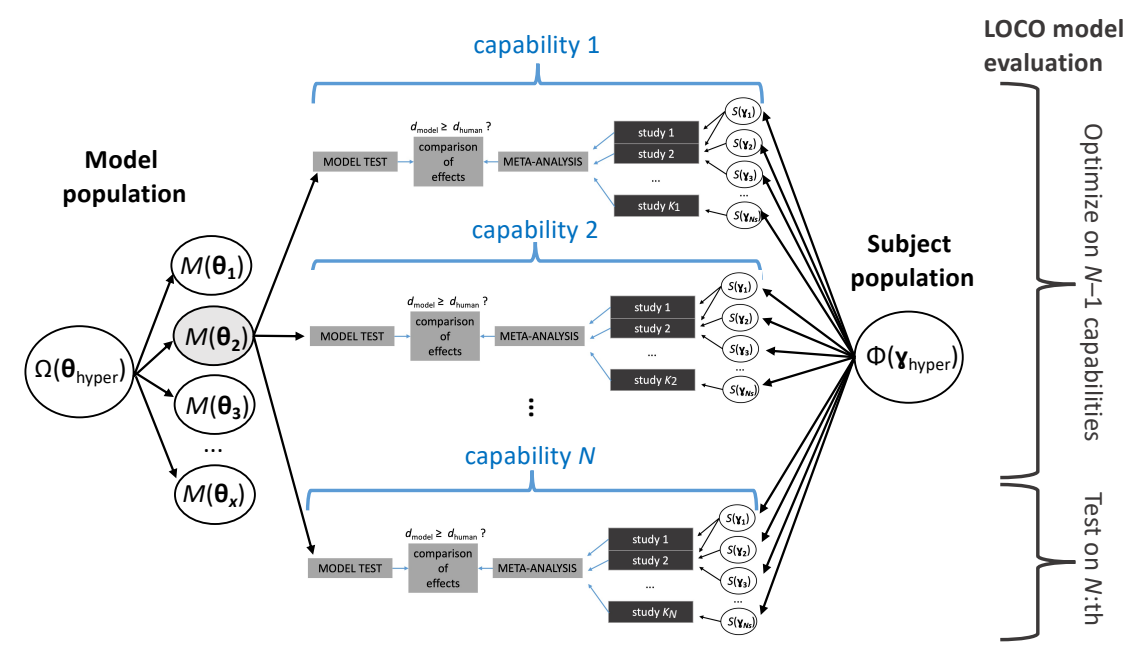

Figure 6: A schematic overview of the proposed evaluation protocol for one model across many language capabilities. See Fig. 1 for an enlarged version for the detailed steps in testing each capability.

Besides providing a richer view of model behavior and hence enabling the development of more holistic models, evaluation across multiple capabilities provides yet another benefit: added protection against overfitting to human data. The reference data derived from a single meta-analysis for one linguistic capability is relatively low-dimensional (essentially the main average effect + the effects as 
a function of key moderators, primarily age). Although a representative and rich computational test for the given capability (with hundreds or thousands of test trials) with proper effect size calculation already protects against trivial solutions that could produce the correct outcome patterns, there is still a real risk of model overfitting to the data. This is due to the large number of design choices and hyperparameters associated with typical computational models operating on real speech data (see also Yarkoni and Westfall, 2017). The overfitting can take place by accident, by deliberate (but benevolent) exploration of model designs and hyperparameters until the correct output pattern emerges, or by global optimization of model design and hyperparameters using the human data as the optimization target (e.g., Snoek et al., 2015 and references therein). In contrast, when evaluating against multiple capabilities in parallel, the risk of coming up with models with zero real-life explanatory value decreases as more capabilities get tested.

Furthermore, in order to enable global optimization with respect to human data, we propose a so-called leave-one-capability-out (LOCO) protocol for computational model evaluation. In LOCO, the model and its hyperparameters can be optimized using all but one capability in the used evaluation test battery, after which the optimized model is tested on the held-out capability. The process is repeated until all capabilities of interest have been used as the held-out test data. Since the optimization process does not get any feedback for the tested capabilities, a model providing a good fit across all the test folds can be considered as a strong one, generalizing to explain language capabilities outside the scope of its optimization process. However, given the complexity of typical models and the extent of computation involved in simulating language learning from low-level sensory percepts, LOCO-based model optimization may not be trivial to perform in practice even with today's advanced computing hardware, and without forgetting sustainability considerations associated with large-scale scientific computing (cf., e.g., García-Martín et al., 2019). On the other hand, countless training/testing/improving-iterations on "large-enough" data are a typical part of computational model development, and therefore principled data-driven approaches that replace ad-hoc tests in optimization of unknowns can also contribute to more sustainable research practices in the long run.

Guideline \#8: Testing a model across multiple linguistic capabilities reduces the risk of overfitting to human data and is needed in the development of more holistic models of child language development.

Guideline \#9: Leave-one-capability-out (LOCO) evaluation can be applied for principled model optimization with a reduced risk of overfitting to the phenomena of interest.

Finally, since creation of representative and flexible computational tests for new language capabilities is by no means trivial in terms of design and effort, it would benefit scientific progress if researchers would co-operate on establishing and further developing model testing protocols. This would complement the efforts on integrated collaborative data through platforms such as MetaLab and Wordbank (Frank, Braginsky, et al., 2017; Lewis et al., 2016). While this comes with practical challenges related to open sharing of audio stimuli, we believe that these hindrances are outweighed by the increased replicability and scalability of future modeling efforts. Hence, the final guideline recommends open dissemination of test protocols for computational models.

Guideline \#10: Linguistic capability tests for computational models should be openly documented and shared to facilitate collaborative science.

\section{Experiments}

The goal of our experiments is to illustrate the meta-analytic model evaluation approach in two language capabilities: infant-directed speech preference and native and non-native vowel discrimination. LOCO evaluation is not demonstrated due to the current lack of sufficiently many computational 
tests for different language capabilities and due to lack of computationally feasible test-based model optimization practices for the current models of interest.

\subsection{Studied models}

We chose to study two popular and recently-proposed self-supervised models of speech representation learning in our experiments, namely Autoregressive Predictive Coding (APC; Chung et al., 2019), and Contrastive Predictive Coding (CPC; Oord et al., 2018). They are both so-called predictive coding models, where the learning criterion is based on minimizing prediction error of future observations (cf. the concept of predictive inference in neuroscience; e.g., Friston, 2005, 2010). They are both deep neural network models. The basic difference between APC and CPC is the type of targets they are predicting: APC focuses on predicting future speech observations $\mathbf{x}(t+k)$ (e.g., spectral feature vectors extracted from the waveform; hence vector notation for $\mathbf{x}$ ) at a temporal distance $t+k$, given the observations $\mathbf{x}(0), \ldots, \mathbf{x}(t-1), \mathbf{x}(t)$ up to $t$. APC learning process then attempts to optimize the parameters $\boldsymbol{\theta}$ of the predictor model $M_{\mathrm{APC}}(\boldsymbol{\theta})$ such that the prediction errors (L1 distance between true and predicted future observations) gets minimized on the training data. CPC, in contrast, uses an encoder network to extract latent representations $\mathbf{z}(t)=f(\mathbf{x}(t))$, and then a predictor network attempts to predict future latents $\mathbf{z}(t+1), \mathbf{z}(t+2), \ldots, \mathbf{z}(t+k)$ up to $t+k$ from past latents $\mathbf{z}(t-\infty), \ldots, \mathbf{z}(t-1), \mathbf{z}(t)$ such that the process maximizes the probability of true future latents while minimizing the probability of randomly sampled "false future latents" (negative samples) $\mathbf{z}_{\text {neg }}(t+r)$, where $r \in \mathbb{Z}$ with the constraint $r \notin\{1,2, \ldots, k\}$. The learning process of $M_{\mathrm{CPC}}(\boldsymbol{\theta})$ consists of jointly learning the latent space encoder for $\mathbf{z}$ and the predictor network responsible for predicting the future latents, as optimized using Noise Contrastive Estimation (Gutmann and Hyvärinen, 2010). Both APC and CPC and their modifications have shown to be promising unsupervised learning approaches for different linguistic representations in different speech tasks (e.g., phone classification: Chung et al., 2019; Oord et al., 2018; phone segmentation: Kreuk et al., 2020; and word segmentation Bhati et al., 2021; see original papers for methodological details).

In both of our experiments, input features $\mathbf{x}$ to APC and CPC consisted of 39-dimensional Melfrequency cepstral coefficient (MFCC) spectral feature vectors extracted every 10-ms with a window of 25-ms. APC was implemented as a three-layer multilayer perceptron (MLP) with rectified linear unit activations, 128 units each (with droput of 0.2 ), followed by three layers of gated recurrent units (GRUs), connected with residual connections, and each GRU layer consisting of 512 units. APC used prediction distance of $k=5$ frames $(50 \mathrm{~ms})$ found suitable for phonemic learning in earlier work (e.g., Chung et al., 2019). CPC encoder was implemented as an MLP with 3 layers of 512 ReLu units and dropout of 0.2 , whereas the CPC predictor network was a GRU layer with 256 units. CPC used a maximum prediction distance of $k=12$ frames, also based on earlier literature (Oord et al., 2018), and the latent space dimensionality was set to 512 . The models were used to simulate English monolingual learners by training them on the 100-hour subset of LibriSpeech corpus (Panayotov et al., 2015). Training was continued until APC/CPC loss on a Librispeech validation set (dev-librispeech) failed to improve for 5 epochs (for APC) or 10 epochs (for CPC), as based on the findings by Cruz Blandón and Räsänen (2020). To model a "newborn" infant, we also evaluate APC and CPC with a set of randomly initialized parameters before any training on speech data.

For extraction of $\mathbf{z}(t)$ during the computational tests, for CPC we simply use the latents extracted by the CPC encoder. For APC, we use the latent representation from the last GRU layer of the predictor network (see Chung and Glass, 2019). For attentional preference score $a(t)$, we extract model prediction loss values for each input frame. For CPC we use the InfoNCE loss (see Oord et al., 2018) and for APC, we use mean absolute error between the predicted and true future speech 
observation. The larger the loss the more difficult the prediction task is for the model; put otherwise, the lower the loss, the higher the prediction accuracy, which may be likened in some sense to greater familiarity.

All program code for the below-reported experiments, associated models, and language capability tests are available for download at https://github.com/SPEECHCOG/metaeval_experiments/. The Isolated Vowel Corpus used in Experiment \#2 is available at https://github.com/SPEECHCOG/ isolated_vowels_corpus/.

\subsection{Capability \#1: infant-directed speech preference}

As the first linguistic capability, we focus on testing models in infant-directed speech (IDS) preference. IDS refers to a speaking style in which speakers use higher pitch, exaggerated intonation contours, and shorter utterances compared to regular adult-directed speech (ADS) when addressing young children (Fernald et al., 1989; Soderstrom, 2007). In this context, IDS preference refers to the finding that infants seem to prefer listening to IDS over ADS (Fernald, 1985). More broadly, it has been suggested that besides enhancing infant attention towards spoken language, IDS promotes infant-caregiver social interaction, potentially by communicating positive affect, and helps to highlight certain aspects of the speech stream over others for communicative purposes (e.g., Golinkoff et al., 2015; Soderstrom, 2007).

Recently, infant preference towards IDS has been documented in a large-scale replication study across several labs (Frank et al., 2020; see next sub-section), providing an excellent source of empirical data for meta-analytic evaluation. In addition, IDS preference focuses on suprasegmental characteristics of speech in contrast to the second capability to be studied, vowel discrimination, that focuses on segmental characteristics (see Section 4.3). Since IDS preference quantification requires measurement of overt attentional preference towards IDS and ADS stimuli, the test also demonstrates the use of attentional preference scoring mechanism in computational models (see Section 3). Together these factors make IDS preference a feasible setup to start demonstrating the meta-analytic model evaluation practices championed in this paper.

\subsubsection{Meta-analysis of human data}

The most recent meta-analysis of IDS preference was carried out by the ManyBabies consortium (Frank et al., 2020). The ManyBabies project corresponds to a large-scale, multilab study that explicitly investigated replicability of IDS preference experiments by employing the same experimental design and stimuli across different participating labs. The only difference was that different labs used head-turn preference procedure, central fixation, or eye-tracking, depending on their facilities. We have chosen to use this meta-analysis because it is a highly systematic large-scale meta-analytic study on the topic (Guideline \#1), is recent (Guideline \#3), the original speech stimuli together with the meta-analytic methods and data are openly available ${ }^{10}$, and the experimental setup allows straightforward computational test adaptation that can also be openly disseminated due to stimuli availability (Guideline \#10). It has several advantages over typical meta-analyses, including that it is more homogeneous than the other extant meta-analysis on this topic (Dunst et al., 2012), and since it was a registered report, there is no margin for reporting biases.

The ManyBabies dataset includes 67 studies (from 67 different labs), 2, 329 full-term monolingual infants from three to 15 months of age, and 12 participant native languages. For our analyses, we used the subset of North American children (1,066 infants). Infants' looking time was measured as the dependent variable. Cohen's $d_{z}$ was calculated for each study after obtaining the mean

${ }^{10}$ https://osf.io/re95x/ 
difference score for paired trials contrasting IDS and ADS stimuli, and as a function of age group (using three-months age bins) (see Fig. 7 for the effect size distribution). A random-effects model was employed to obtain the mean effect size estimate, which yielded $d=0.43(S E=0.05, p<.0001$, $95 \% \mathrm{CI}=[0.33,0.53]))$ as a reference point for the models. We also replicated the procedure of Frank et al. (2020) for testing the significance of age as a moderator for the effect size. A linear mixedeffect model was applied using trial type, method, trial number and age as moderators in predicting the logarithm of individual looking times from individual participants (as available in ManyBabies study). This analysis revealed a significantly increasing looking time for IDS trials with increasing infant age $(0.016, p<.0001)$. Still following Frank et al. (2020), we derived the developmental effect size trajectories for model comparison by fitting a linear model to age-binned effect sizes obtained from the individual studies (Fig. 9b).

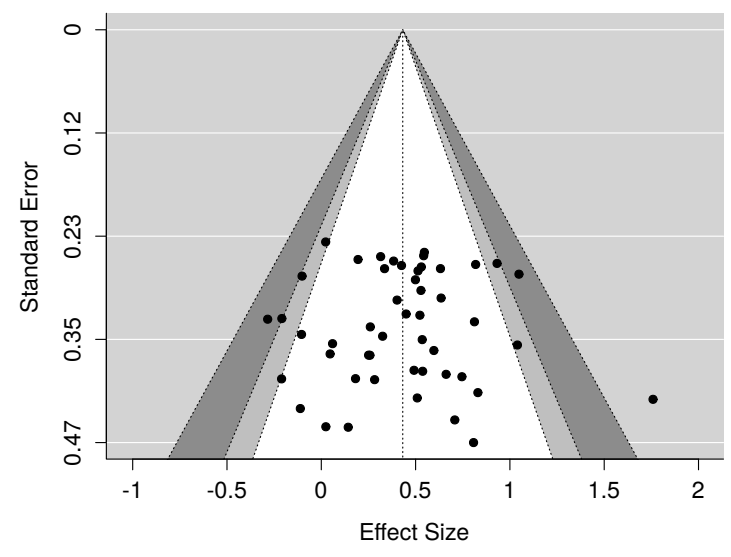

Figure 7: Funnel plot of effect size distribution of infant direct speech preference. The dotted vertical lines mark the weighted effect size estimate $(d=0.43)$. The coloured regions denote the confidence intervals $90 \%$ (white), $95 \%$ (light grey), and 99\% (dark grey).

\subsubsection{Model test setup}

Since this ManyBabies experiment was specifically designed as a representative test for measuring IDS preference cross-linguistically (Frank et al., 2020), we adapted the same setup as our representative computational test with minor modifications (cf. Guideline \#4). In Frank et al. (2020), the stimuli were based on a screened set 120 IDS and 120 ADS utterances spoken in North American English and rated for their IDS- and ADS-likeness using Amazon Mechanical Turk. Utterance duration ranged from $1.3 \mathrm{~s}$ to $23.7 \mathrm{~s}$ (ADS utterances mean duration: $5.1 \pm 2.9 \mathrm{~s}$; IDS utterances mean duration: $2.9 \pm 1.0$ s; see Frank et al., 2020 for recording setup details). A subset of these utterances were presented to infants in eight paired trials, where each IDS (ADS) stimulus in each trial consisted of a 18-s long clip created by concatenation of multiple IDS (ADS) utterances together while balancing for lexical contents of the stimuli across the speaking styles for each trial.

In our setup, we used the same original set of 120 IDS and 120 ADS utterances to extract the attentional preference scores $a(t)$ from tested models (see Section 3). However, since eight paired trials constitutes a rather small number of trials to test a single model, and since we can extract 
model responses already from individual utterances ${ }^{11}$, our setup uses the individual IDS and ADS utterances as trials for the two respective conditions (120 per condition). Consequently, the effect size can be calculated as the standardised mean gain between the mean trial response from IDS trials and the mean trial response from ADS trials. To calculate the effect size, first a trial response is estimated for each trial $i$ :

$$
\alpha_{i}=\frac{1}{T_{i}} \sum_{t=1}^{T_{i}} a(t)
$$

where $T_{i}$ is the total number of timesteps for the trial $i$. Then the mean trial response for the set $V_{\text {IDS }}$ of IDS trials is estimated as

$$
\mu_{\mathrm{IDS}}=\frac{1}{\left|V_{\mathrm{IDS}}\right|} \sum_{i=1}^{\left|V_{\mathrm{IDS}}\right|} \alpha_{i} \quad \mid v_{i} \in V_{\mathrm{IDS}}
$$

and the standard deviation as

$$
\sigma_{\mathrm{IDS}}=\sqrt{\frac{\sum_{i}\left(\alpha_{i}-\mu_{\mathrm{IDS}}\right)^{2}}{\left|V_{\mathrm{IDS}}\right|-1}} \quad \mid v_{i} \in V_{\mathrm{IDS}}
$$

Similarly, $\mu_{\mathrm{ADS}}$ and $\sigma_{\mathrm{ADS}}$ are calculated for the set of ADS trials $V_{\mathrm{ADS}}$. The effect size is then estimated as the standardized mean gain using $\mu_{\mathrm{IDS}}, \mu_{\mathrm{ADS}}$, and the variance for each type of speech as:

$$
d=\frac{\left|\mu_{\mathrm{IDS}}-\mu_{\mathrm{ADS}}\right|}{\sqrt{\frac{\sigma_{\mathrm{IDS}}^{2}+\sigma_{\mathrm{ADS}}^{2}}{2}}} .
$$

\subsubsection{Results}

Before proceeding to the actual model evaluation, we conducted two sanity checks for the computational test. First, we verified that both models yielded null effects for simulated random responses $a(t)$. Second, we assessed that there were large effects for preferences sampled from distinct manuallyspecified parametric distributions for the IDS and ADS trials, we proceeded to benchmark the APC and CPC models against the human data.

\footnotetext{
${ }^{11}$ The need for longer stimuli largely originates from robust measurement of looking time with infants.
} 


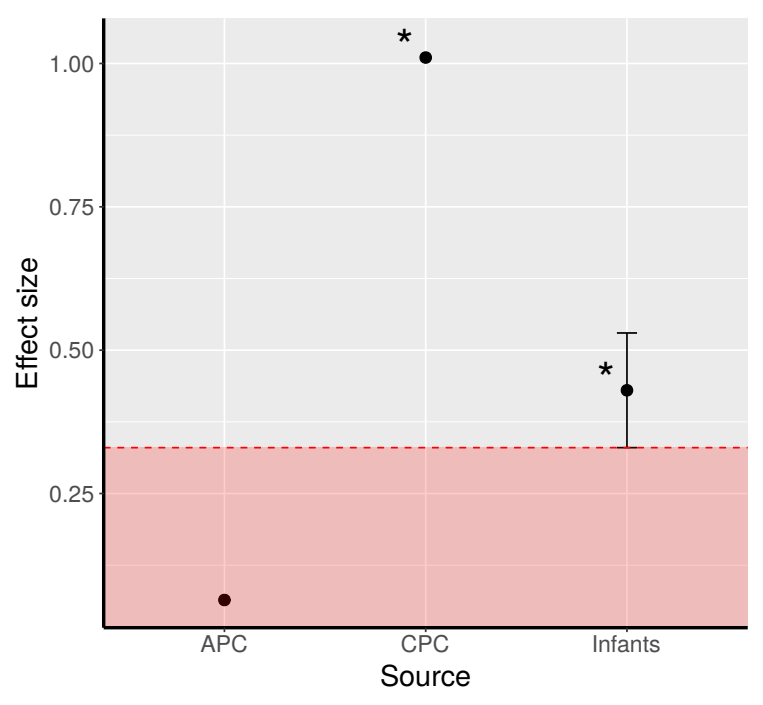

Figure 8: Effect sizes of APC and CPC models and weighted effect size from meta-analysis for the IDS preference. The error bars in the infants' effect size indicates the $95 \%$ confidence interval of the mean effect size estimate. The red region delimits the non-compatible zone for models' effect sizes with human reference effect. Results with a statistically significant difference in preference towards IDS versus ADS (at $p<0.05)$ are marked with *. Note that the effect size estimates for models are point estimates without uncertainty involved (Section 3.4).

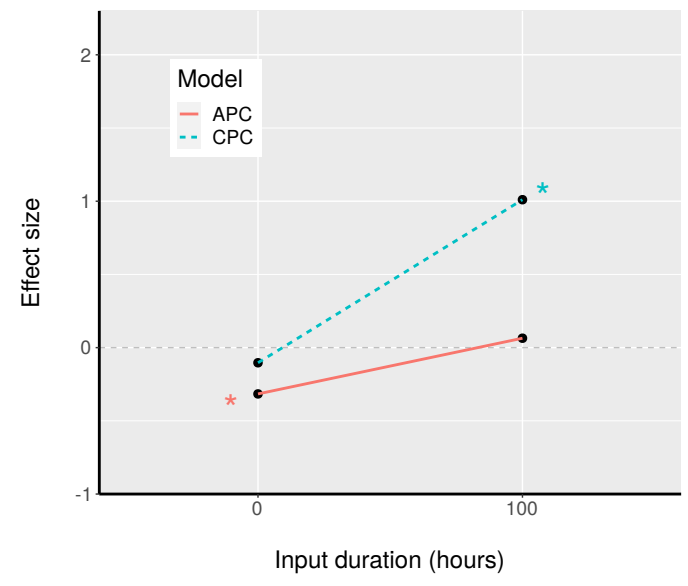

(a) IDS preference: Models

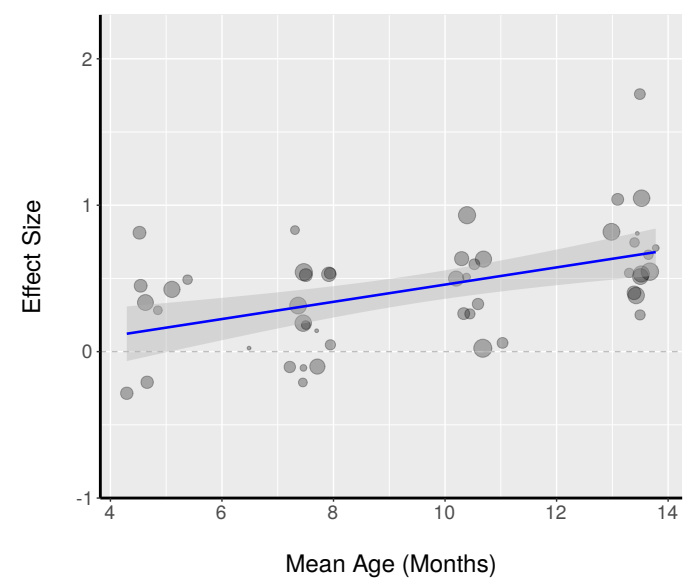

(b) IDS preference: Infants

Figure 9: Effect sizes of APC and CPC models as a function of input duration (amount of speech hours) and effect sizes of infants' data as a function of age. 0 hours of input corresponds to untrained models and 100 hours to trained models. The size of the circles in the infant data represents the sample size of the studies. The APC model is represented with a solid red line and the CPC model with a dashed blue line. Results with a statistically significant difference in preference towards IDS versus $\operatorname{ADS}($ at $p<0.05)$ are marked with *. 
Fig. 8 shows the effect sizes obtained by the models and the reference mean effect size from the infants' meta-analytic data. The effects for IDS preference were $d=0.06, p=0.62$ for the APC model and $d=1.01, p<.0001$ for the CPC model. As calculated in Section 4.2.1, the weighted effect size for infants is $d=0.43,95 \%, C I[0.33,0.53]$. Therefore, by Guideline \# 7 , the APC model's effect is not compatible with the human reference, as it is smaller than the lower limit of the confidence interval of the infants' effect. In contrast, the effect of the CPC model is compatible with that of the infants as it is greater than the indicated confidence interval.

For the developmental trajectory comparison, we compared the model effects without any training and after training (100 h of speech; Guideline \#6). Fig. 9a shows the effect obtained for untrained $(0 \mathrm{~h})$ and trained $(100 \mathrm{~h})$ models while Fig. $9 \mathrm{~b}$ shows the developmental curve estimated for the infants. The plot shows that, although both models' effects increased after training, the APC model actually becomes indifferent to IDS and ADS in terms of preference $(p>0.05)$, whereas CPC effect increases substantially. Given the infant trajectory, the results from models suggest that only the CPC model has a compatible trajectory. Effect sizes of the APC model are not compatible with infants' effects for neither 0 or 100 hours of speech input.

\subsubsection{Discussion for experiment \#1}

The first experiment demonstrates how attentional preference scores derived from computational models can be compared to effect sizes observed from infant literature. It also shows how developmental trajectories of infants can be compared to effect size change in models, as the models get more experienced with language input (i.e., trained with more data). Even though the present meta-analytic evaluation approach does not allow derivation of upper bounds for model effect sizes that would still be compatible with the human data, the experiment already reveals that one of the models, APC, does not appear to be compatible with the IDS preference observed in real infants. In contrast, preference exhibited by the CPC model appears to be compatible with the human data.

The experiment is primarily intended to showcase the basic idea of our evaluation approach, and should not be taken as comprehensive analysis of APC and CPC as models of infant learning. To begin with, the test included only two "developmental" checkpoints for models, one without any training and one with $100 \mathrm{~h}$ of speech to learn from. Moreover, the attentional preference mechanism was implemented in both models simply as the output of the loss function. This simulates full attentional focus towards less predictable inputs ("novelty preference"). In practice, the dependency between stimulus predictability and infant attentional preference is not monotonic with respect to surprisal in input (e.g., Hunter and Ames, 1988; Kidd et al., 2012). These preference mechanisms could therefore also be modeled in many ways, operating on top of the basic self-supervised learning algorithms that do not aim to simulate human preferences as a part of their basic functionality (Chung et al., 2019; Oord et al., 2018). Therefore, the present experiment should not be considered as the ultimate say on the compatibility of the tested models with respect to human data, but as an illustration of how such a comparison could be conducted in the first place.

The experiment also revealed certain properties of the models and IDS/ADS characteristics. For instance, the APC model showed an ADS preference before observing any speech $(d=-0.32, p=$ 0.02), while the CPC model obtained a null effect in the same situation. This discrepancy is likely due to how these models operate. The APC model is built to predict the input speech features that will occur some tens of milliseconds later from the so-far observed input. Without any training, this prediction task can be considered as easier when the input signal changes more slowly or has longer stable periods. Indeed, in the IDS stimuli, the speaking rate is slower and the utterances are shorter. On the other hand, the CPC model focuses on discriminating between self-learnt representations (or latents, see Section 4.1). Therefore, before training, the model has no assumptions on how those 
latents should be, yielding equality for ADS and IDS in the prediction task $(d=-0.10, p=0.42)$.

\subsection{Capability \#2: vowel discrimination}

As the second capability, we exemplify our approach with native and non-native vowel discrimination. Vowel discrimination refers to the perceptual ability to differentiate between vowels of contrasting phonemic categories. Vowel discrimination is one of the first linguistic capabilities that infants exhibit regarding sound segments (Fig. 1 in Dupoux, 2018). There is evidence of vowel discrimination in infants as young as two months of age (e.g., Marean et al., 1992; Miller and Eimas, 1979; Trehub, 1973). Hence, vowel discrimination is a natural choice for capabilities to be tested with the evaluation protocol.

Typically, experimenters test this capability by presenting two conditions to the infant (same and different perceptual vowels) and measuring a dependent variable (e.g., looking time) to detect possible changes between the two conditions, indicating discrimination capability. Typical methods used to assess vowel discrimination include behavioral paradigms such as Central Fixation (CF; Mugitani et al., 2009) and Conditioned Head-Turn (CHT; Kuhl, 1979), or neural response analysis with EEG using the so-called oddball paradigm (Cheour et al., 1998) or using stimulus alternation with Near-Infrared Spectroscopy (NIRS; Minagawa-Kawai et al., 2007). This line of research has produced different theoretical frameworks to explain how infants become more accurate in discriminating vowels in their native language repertoire and/or less accurate for non-native vowels, a phenomenon that is called perceptual attunement (considered in, e.g., the optimality theory for phonological acquisition by Kager et al., 2004, the exemplar theory by Pierrehumbert, 2003, native language magnet theory by Kuhl et al., 2008, and the developmental framework PRIMIR described in Werker and Curtin, 2005).

Hereafter we describe how we created a computational test for vowel discrimination and tested $\mathrm{APC}$ and $\mathrm{CPC}$ models using it.

\subsubsection{Meta-analysis of human data}

There is no dataset similar to the ManyBabies one studied in the first experiment. Therefore, we chose MetaLab as our source of meta-analytic data and methods for the vowel discrimination task. Using meta-analyses that are based on published literature allows us to illustrate some additional complexities in the approach. To begin with, on MetaLab, the vowel discrimination task is split into two meta-analyses: native and non-native vowel contrasts based on meta-analysis reported by Tsuji and Cristia (2014). In their meta-analysis, Tsuji and Cristia investigated vowel discrimination for the infant age range of $0-15$ months. Following their inclusion criteria and outliers policy, the final subset of effects includes infants with seven different native languages and six experimental methods. The native contrasts subset contains 101 effects, and the non-native contrasts subset contains 31 effects. ${ }^{12}$ Figs. 10a and 10b show the funnel plots of the effect size distribution for native and non-native vowel contrasts. Funnel plots are helpful to represent heterogeneity in effect sizes and their errors, for instance that emerging from publication bias. Both plots display an asymmetry in the distribution of the effects, which is consistent with publication bias: Effect size being correlated with error could indicate that studies that have smaller effect sizes are only published when they have very large sample sizes (and small errors); and/or that a bias for significant effects leads to the selective publication of studies that happen to have a large effect merely because they are less precise,

${ }^{12}$ Datasets downloaded on 01.03.2021 for native contrasts and 18.03 .2021 for non-native contrasts. 
and thus they can over-estimates the true underlying effect. ${ }^{13}$ Note that asymmetries are hard to interpret in the face of high heterogeneity, which is the case here (e.g., six different experimental methods are used).

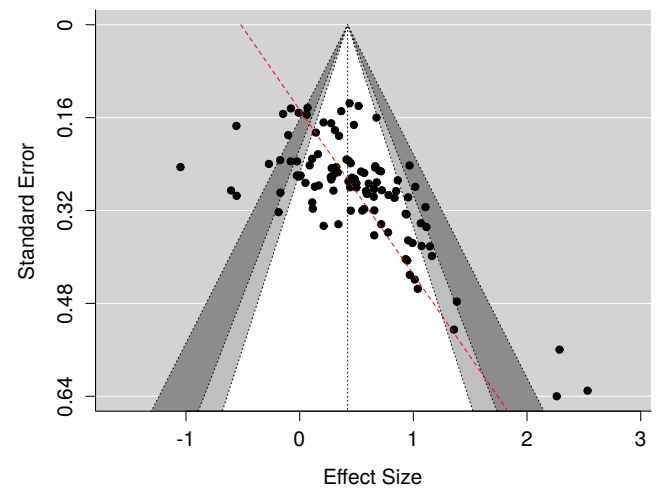

(a) Native vowel contrasts

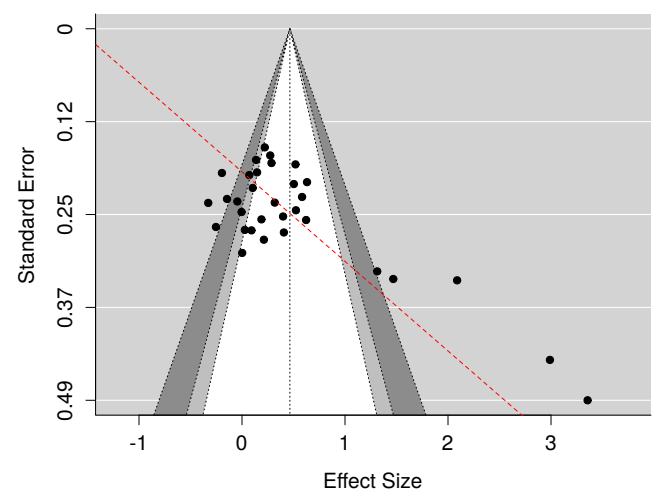

(b) Non-native vowel contrasts

Figure 10: Funnel plots of effect size distribution for native and non-native vowel contrasts. The dotted vertical lines mark the weighted effect size estimate $(g=0.42$ for native contrasts and $g=0.46$ for non-native contrasts). The coloured regions denote the confidence intervals $90 \%$ (white), 95\% (light grey), and 99\% (dark grey). The red dashed lines shows the regression fit.

Following Tsuji and Cristia (2014), a random-effects model with Hedges' effect sizes (g) was employed for the analysis due to the small sample size of some of the included studies. The analysis showed positive effects for the discrimination capability for both contrasts, which will be used to compare the computational models' effects. The estimate for weighted effect sizes for native and non-native vowel contrasts are statistically different from zero with $g=0.42$ for native contrasts $(S E=0.04, p<.0001,95 \% \mathrm{CI}=[0.33,0.51])$ and $g=0.46$ for non-native contrasts $(S E=0.13, p=$ $0.0003,95 \% \mathrm{CI}=[0.21,0.72])$.

The analysis of the effect size as a function of age was carried out employing a meta-regression analysis following MetaLab's up-to-date moderators analysis. As moderators, the regression included age, response mode (e.g., behavior), and exposure phase (e.g., habituation). In comparison with Tsuji and Cristia (2014), where they found a significant age estimate only for native contrasts, the predictors did not reach significance for age estimates on either contrast type with current data. This indicates that age as a moderator does not account for the variability in the effect size distribution with the current data. While this is in contrast with the common understanding on perceptual attunement in early development (e.g., Kuhl et al., 2008), we treat this as the empirical reference point for the phenomenon, as grounded on an up-to-date collection of relevant studies and metaanalytic approach based on earlier literature (Tsuji and Cristia, 2014). Considering these results, the developmental target for the models corresponds to a minimum effect size level that does not change with more language experience, and where the minimum effect compatible with human data is given by the lower bound of the infant effect size confidence interval (Guideline \#7) .

\footnotetext{
${ }^{13}$ This visual inspection was confirmed with Egger's test that obtained intercepts significantly different from zero and significant Kendall's $\tau=0.42, p<.0001$ for native contrasts and $\tau=0.27, p=0.03$ for non-native contrasts.
} 


\subsubsection{Model test setup}

Different to the IDS preference capability, the empirical data for vowel discrimination included studies with a wide variety of methodologies and experimental setups. As indicated by Guideline \#4, the computational test should be representative. To meet this requirement, we reviewed the methods and vowel contrasts used in infants' experiments in order to adapt the key properties to the computational test (see Table 3 for references). We also accounted for test flexibility (Guideline \#5) by focusing on a test that does not involve further model training/adaptation during the test, hence excluding any "habituation" approaches for the computational testing.

The shared characteristic between all different experimental paradigms with infants is to probe for a difference in perceptual encoding of vowel tokens from different phonemic categories, and to contrast this with encoding of vowel tokens from the same category. Therefore, we developed a test where we directly extract the encoded latent stimulus representation $\mathbf{z}(t)$ for each vowel, and then calculate the distances of the representations between categorically equivalent and different vowels. If the model has learnt to discriminate vowels, we expect the distance between representations of categorically equivalent vowels to be lower than between categorically different vowels. Thus, using the distance measure as the dependent variable, a model's vowel discrimination capability can be evaluated (see also Schatz et al., 2013; Schatz et al., 2014 for a similar approach).

Two trial sets of vowel stimuli were defined for model effect size estimation: condition A for categorically distinct vowels $V_{A}$, and condition B for vowels of the same category $V_{B}$. In condition A, each trial $v_{i}^{A} \in V_{A}$ was defined as a pair of speech stimuli $\left\{\mathbf{x}_{j}, \mathbf{x}_{k}\right\}$ corresponding to different vowels with pre-defined contrasts (see below) and from different speakers:

$$
v_{i}^{A}=\left\{\mathbf{x}_{j}, \mathbf{x}_{k}\right\} \mid j \neq k, \operatorname{cat}\left(\mathbf{x}_{j}\right) \neq \operatorname{cat}\left(\mathbf{x}_{k}\right), \text { speaker }\left(\mathbf{x}_{j}\right) \neq \operatorname{speaker}\left(\mathbf{x}_{k}\right)
$$

where $\operatorname{cat}(\mathbf{x})$ stands for vowel category of speech stimulus $\mathbf{x}$ while speaker $(\mathbf{x})$ is identity of the speaker (or voice for synthetic data; see below), and so that for each $i$ a unique tuple $\{j, k\}$ was assigned (i.e., forming across-contrast pairs across all potential combinations). For condition B with the same vowel category, the process was identical except that the vowel category was the same for both stimuli:

$$
v_{i}^{B}=\left\{\mathbf{x}_{j}, \mathbf{x}_{k}\right\} \mid j \neq k, \operatorname{cat}\left(\mathbf{x}_{j}\right)=\operatorname{cat}\left(\mathbf{x}_{k}\right), \text { speaker }\left(\mathbf{x}_{j}\right) \neq \operatorname{speaker}\left(\mathbf{x}_{k}\right)
$$

Responses to both type of trials were defined using DTW total path cost with cosine distance (Appendix A):

$$
\alpha_{i}=D T W\left(\mathbf{z}_{j}, \mathbf{z}_{k}\right)=\sum_{t \in \hat{T}_{i}} 1-\frac{\hat{\mathbf{z}}_{j}(t) \cdot \hat{\mathbf{z}}_{k}(t)}{\left\|\hat{\mathbf{z}}_{j}(t)\right\|\left\|\hat{\mathbf{z}}_{k}(t)\right\|}
$$

where $\hat{\mathbf{z}}(t)$ stands for DTW-aligned latent representations extracted from $\mathbf{x}(t)$ and $\hat{T}_{i}$ is the total duration of the aligned pair (in frames).

Hence, the cross-category mean distance was defined as

$$
\mu_{A}=\frac{1}{\left|V_{A}\right|} \sum_{i=1}^{\left|V_{A}\right|} \alpha_{i} \quad \mid v_{i} \in V_{A}
$$

and cross-category variance as

$$
\sigma_{A}^{2}=\frac{1}{\left|V_{A}\right|-1} \sum_{i=1}^{\left|V_{A}\right|}\left(\alpha_{i}-\mu_{A}\right)^{2} \quad \mid v_{i} \in V_{A} .
$$


Within-category $\mu_{B}$ and variance $\sigma_{B}^{2}$ were calculated in an analogous manner from all $v_{i} \in V_{B}$. Then the effect size can be calculated as the standardized mean gain same as in IDS preference (Eq. ( 15)). Since the infant meta-analysis uses Hedges' g, we converted model d to Hedges' g for model and human comparison (Hedges and Olkin, 1985). For this test, we utilized data from multiple speech corpora, as explained in the next sub-section, and therefore the effect sizes were first calculated per corpus and contrast before averaging them to obtain the final effect size estimate.

Stimuli We used three sources of audio for the vowel stimuli: 1) English vowels embedded in /CVC/ contexts from the Hillenbrand vowel database (Hillenbrand et al., 1995) to simulate native contrasts for our English-trained models, 2) non-native contrasts embedded in /CVC/ contexts from the German OLLO logatome speech database (Meyer et al., 2010), and 3) isolated vowels /V/ synthesized with MBROLA speech synthesizer (Dutoit et al., 1996), a widely utilized system in previous behavioral research, to simulate both native and non-native contrasts. Hillenbrand corpus contains 12 American English vowels embedded in /hVd/ context (e.g., /hrd/ as in "head"). Each of the 139 speakers recorded each vowel once. The recordings include male, female and child speakers. OLLO corpus consists of ten German vowels embedded in eight /CVC/ contexts. The recordings include several sources of variability, such as speaking style and repetitions (withinspeaker variability). For our experiments, we used the "OLLO2.0_NO" subset that comprises ten adult speakers with the same accent for a total of 14,347 recordings.

Since synthetically modified stimuli have been employed in infants studies (e.g. Bohn and Polka, 2001), a novel isolated vowels corpus (henceforth IVC) was developed with the MBROLA synthesizer. Using the descriptors of Marean et al. (1992) for vowel duration (500 ms) and pitch contours, we synthesized vowels for English (EN), French (FR), Japanese (JP), Dutch (NL) and German (DE) languages. Each vowel was synthesised with all available voices for each language ${ }^{14}$ and including low and high pitch contours, as specified in Marean et al. (1992), producing a total of 244 recordings. The contrasts were chosen based on those tested in the literature. Table 3 lists the vowel contrasts included in the test. ${ }^{15}$

\subsubsection{Results}

We started with two sanity checks for the computational test. First, we verified that both models yielded a null effect for random signal representations and a very large effect for hand-crafted orthogonal vector representations for different vowel identities. Second, we ensured that both APC and CPC model training had been successful by performing standard supervised frame-level phone classification task on Librispeech corpus (Panayotov et al., 2015) using the latents $\mathbf{z}$ from untrained and trained models as features in the classifier. Untrained models reached phone classification accuracies of $21 \%$ and $28 \%$ for APC and CPC on held out data, respectively, while trained models reached $54 \%$ and $52 \%$. This indicates that the models had learned representations that were sensitive to phonetic structure of the data, as already observed in Oord et al. (2018) and Chung et al. (2019).

As for our main test, Figs. 11a and 11b depict the overall effect sizes obtained by the APC and $\mathrm{CPC}$ models in the vowel discrimination test along with the effect size estimate and its confidence intervals from the meta-analysis. For native contrasts, the model effects were $g=1.84, S D=1.35$ for the APC model and $g=2.27, S D=1.70$ for the CPC model. For non-native contrasts, the effects were $g=0.83, S D=0.95$ for the APC and $g=1.16, S D=1.35$ for the CPC. As derived in Section

\footnotetext{
${ }^{14}$ MBROLA voices available on https://github.com/numediart/MBROLA-voices

${ }^{15}$ For the OLLO, the selected contrasts do not have exact German counterpart in the literature. However, we formed contrasts that were tested for other languages. For the IVC, Japanese and German non-native contrasts were tested for Japanese infants.
} 
Table 3: Native and non-native vowel contrasts included in the test. IVC: Isolated vowels corpus. $\left|V_{A}\right|$ and $\left|V_{B}\right|$ are the total number of trials for the categorically different and same vowel conditions. The total number of unique speakers per contrast is also reported. Sources marked with $(*)$ indicate contrasts that were originally tested with infants for other languages, but were adopted to our experiments using a similar contrast from the included speech corpora.

\begin{tabular}{|c|c|c|c|c|c|c|}
\hline Contrasts & Source & $\left|V_{A}\right|$ & $\left|V_{B}\right|$ & Speakers & Language & Corpus \\
\hline$/ \mathrm{a} /-/ \mathrm{i} /$ & $\begin{array}{l}\text { (Kuhl, 1979; Kuhl } \\
\text { and Miller, 1982; } \\
\text { Marean et al., 1992; } \\
\text { Trehub, 1973) }\end{array}$ & 18,768 & 18,771 & 139 & \multirow{5}{*}{ English } & \multirow{5}{*}{ Hillenbrand } \\
\hline /i/-/I/ & $\begin{array}{l}\text { (Trainor and Des- } \\
\text { jardins, 2002) }\end{array}$ & 19,182 & 19,182 & 139 & & \\
\hline$/ \mathrm{a} /-/ \varepsilon /$ & $\begin{array}{l}\text { Cowan et al., } \\
1982)\end{array}$ & 18,363 & 18,360 & 139 & & \\
\hline $\mid \mathrm{e} /-/ \varepsilon /$ & $\begin{array}{l}\text { (Sundara and } \\
\text { Scutellaro, 2011) }\end{array}$ & 18,768 & 18,771 & 139 & & \\
\hline$/ a /-/ æ /$ & $\begin{array}{l}\text { (Miller and Eimas, } \\
1979 \text { ) }\end{array}$ & 18,227 & 18,225 & 138 & & \\
\hline $\mid \varepsilon /-/ \mathrm{I} /$ & $\begin{array}{l}\text { Mulak et al., } \\
2017)^{*}\end{array}$ & 206,928 & 206,928 & 10 & \multirow{3}{*}{ German } & \multirow{3}{*}{ OLLO } \\
\hline$/ \mathrm{i} /-/ \mathrm{I} /$ & $\begin{array}{l}\text { (Trainor and Des- } \\
\text { jardins, 2002)* }\end{array}$ & 206,928 & 206,928 & 10 & & \\
\hline /a/-/a:/ & $\begin{array}{l}\text { (Minagawa-Kawai } \\
\text { et al., 2007; Mugi- } \\
\text { tani et al., 2009)* }\end{array}$ & 205,635 & 205,635 & 10 & & \\
\hline$/ \mathrm{a} /-\mathrm{i} /$ & (see above) & 24 & 24 & 3 & \multirow{4}{*}{ English } & \multirow{8}{*}{ IVC } \\
\hline /i/-/I/ & (see above) & 24 & 24 & 3 & & \\
\hline $\mid a /-/ \varepsilon /$ & (see above) & 24 & 24 & 3 & & \\
\hline$/ a /-/ æ /$ & (see above) & 24 & 24 & 3 & & \\
\hline$/ \mathrm{a} /-/ \tilde{\mathrm{a}} /$ & (Trehub, 1976) & 168 & 168 & 7 & French & \\
\hline$/ \varepsilon /-/ \mathrm{I} /$ & (Mulak et al., 2017) & 8 & 8 & 2 & Dutch & \\
\hline /a:/-/a/ & $\begin{array}{l}\text { (Minagawa-Kawai } \\
\text { et al., 2007; Mugi- } \\
\text { tani et al., 2009)* }\end{array}$ & 8 & 12 & 3 & Japanese & \\
\hline /u:/-/y:/ & $\begin{array}{l}\text { (Mazuka et al., } \\
2014)^{*}\end{array}$ & 168 & 168 & 7 & German & \\
\hline
\end{tabular}

4.3.1, the corresponding human effects were $g=0.42,95 \% C I=[0.33,0.51]$ for native contrasts and $g=0.46,95 \% C I=[0.21,0.72]$ for non-native contrasts. The effect sizes for both types of contrasts and models are thereby greater than those indicated by the confidence intervals of the mean effect sizes from human data. By guideline \#7, the APC and CPC models' performances are compatible with infant data in the vowel discrimination task. 


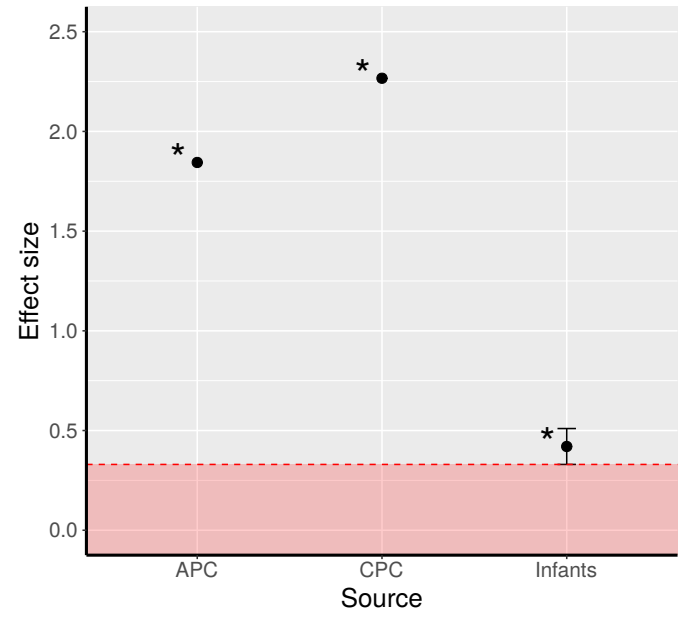

(a) Native vowel contrasts

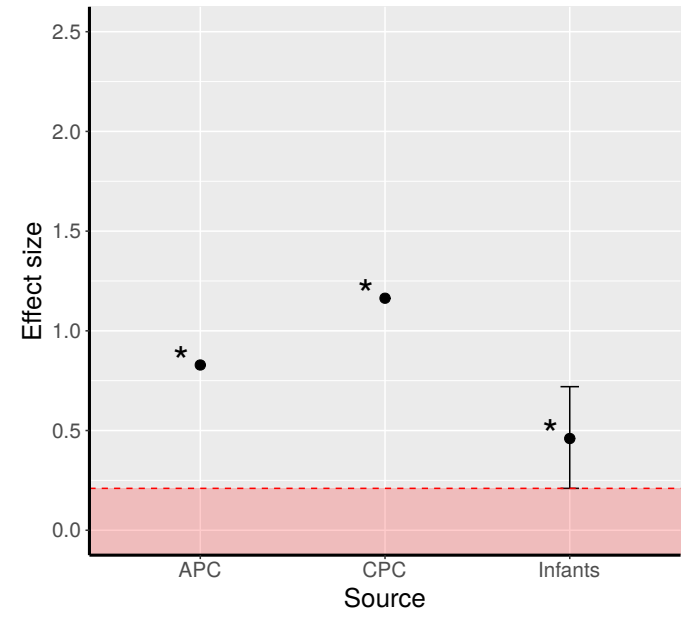

(b) Non-native vowel contrasts

Figure 11: Effect sizes of APC and CPC models and weighted effect sizes yielded by meta-analysis for native and non-native contrasts. The error bars indicate the $95 \%$ confidence interval of the mean effect size for infants' data. The red region indicates the area where model effect size is incompatible with human effect. Significant estimates at $p<0.05$ are marked with *. Since model effect sizes are deterministic point estimates, there is no uncertainty involved with them (Section 3.4). 


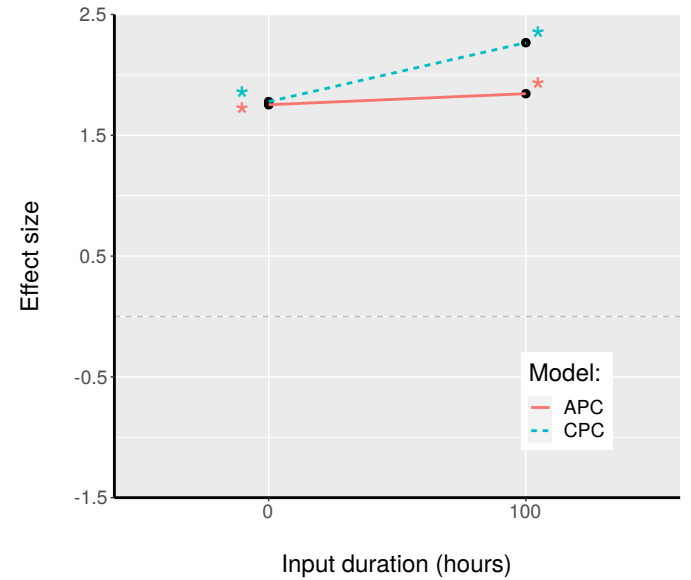

(a) Native contrasts: Models

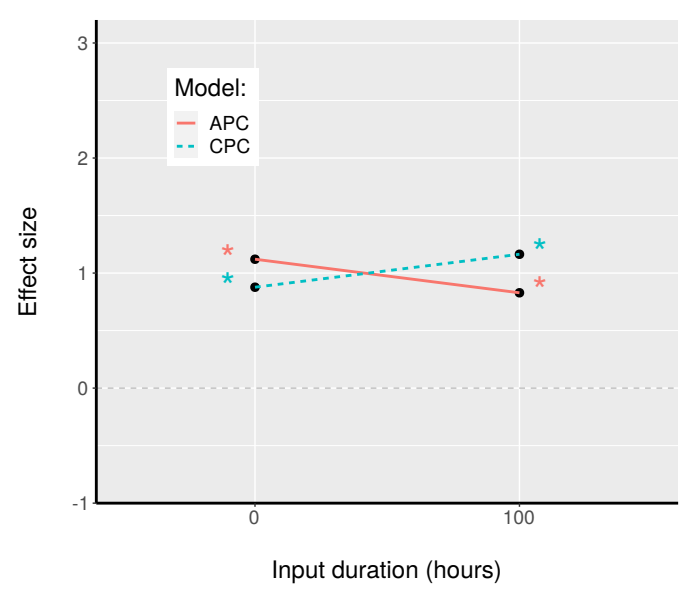

(c) Non-native contrasts: Models

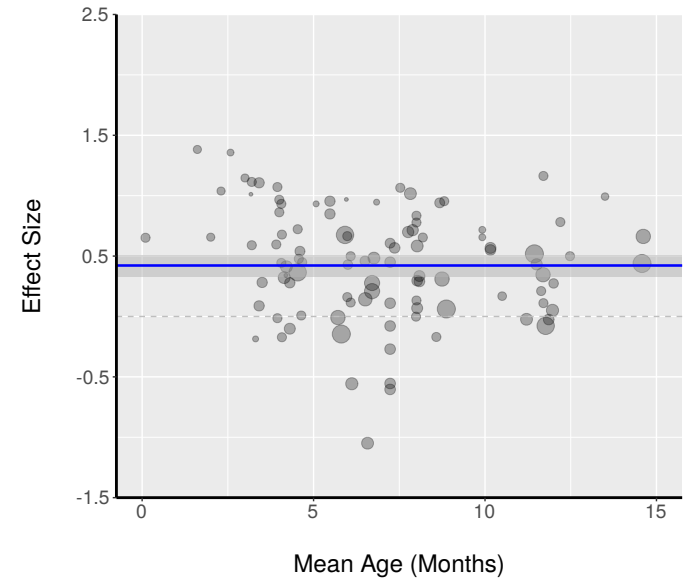

(b) Native contrasts: Infants

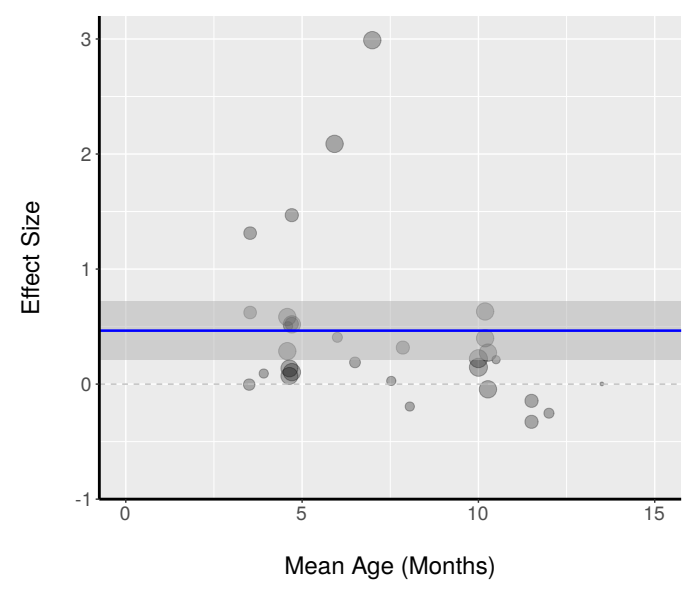

(d) Non-native contrasts: Infants

Figure 12: Effect sizes as a function of input duration (models) and age (infants) for native and non-native contrasts. 0 hours of input corresponds to untrained models and 100 hours to trained models. The size of the circles in the infant data represents the sample size of the studies. Significant effects $(p<0.05)$ are marked with *. 
Similarly to the IDS preference experiment, we also compared untrained and trained models for the change in effect change as a function of language experience (Guideline \#6). Fig 12a shows the effects obtained for the untrained and trained APC and CPC models and for the native contrasts, and $12 \mathrm{c}$ shows the same for non-native contrasts. Lines indicates the direction of change of the effects. The result shows that, for the native contrasts, both the CPC and the APC model showed an increased effect due to training. As for the non-native contrasts, the APC model's effect decreased with training, whereas the CPC model's effect increased. As a reference, Figs $12 \mathrm{~b}$ and $12 \mathrm{~d}$ plot the infant effects size as a function of infant age. Although models have effect sizes compatible with infants' effect with 0 and 100 hours of input, their developmental trajectories are incongruent with infant data due to change with experience. However these results should be taken with a grain of salt, given that our current setup does not reflect the actual range of language experience (in terms of speech hours) available to 0-15 month-old-infants, the age inclusion criterion used in the employed meta-analysis.

\subsubsection{Discussion for experiment \#2}

The second experiment demonstrated how internal states of computational models can be mapped to measurable effect sizes from a vowel discrimination task, and to what degree these effects can then be compared to the effect sizes observed in behavioral data. Again, the meta-analytic approach enables derivation of lower bounds for the required model effects, whereas upper bound of yet compatible effect sizes is not accessible with the present means (Section 3.4).

The experiment showed that the two predictive coding models learnt speech representations that show an effect size compatible with the mean effect size exhibited by infants. At first, this might be interpreted as if the predictive mechanisms underlying learning in APC and CPC lead to performance compatible with infant data. However, since the effect size is already relatively large before the models have seen any training data, and since the changes in the overall effect size due to training are quite modest compared to the starting point, it appears that the predictive mechanisms of these models are not the primary reason for the observed findings. Instead, the capability to distinguish different vowels from each other is already expected on the basis of standard time-frequency analysis of speech, as in this case captured by the MFCC features used as inputs to the models. Technically speaking, the neural network layers of the untrained models correspond to a series of non-linear transformations of the input in high-dimensional spaces, where these transformations are determined by the randomly initialized parameters of the models. Since the transformations are deterministic (given the initial parameters), they are also likely to approximately preserve similarity properties of the MFCC input space. In other words, inputs that are similar in the MFCC domain are also likely to be similar in the latent encoding space, whereas inputs that are distant in the input space are also likely to be distant in the latent space before any learning has taken place. Since MFCCs already carry phonemic information while ignoring some non-phonemic variability (e.g., F0) due to their design, the corresponding latent encodings are also likely be discriminative with respect to vowel categories (see also, e.g., Chrupała et al., 2020; Khorrami and Räsänen, 2021). In this context, it appears that much of the vowel discrimination is already explainable in terms of the input features, but the discriminatory characteristics of the latents then change somewhat as the models learn from input data: $\mathrm{CPC}$ improves on both native and non-native contrasts, while APC is relatively stable on the native contrasts and degrades on the non-native ones.

One property of the present test is that, in order to develop a computational test as close as those presented to infants, we employed logatome and CVC corpora to extract a number of vowel contrasts earlier utilized in the behavioral literature. However, developing a balanced setup (in terms of identity and quantity of contrasts for native and non-native vowels) is subject to the availability 
of such corpora and the types of contents in each of the corpora. This leads to practical constraints in terms of how many tokens are available for each vowel, how rich phonetic contexts they occur in, and how well different speakers and their voices are represented in the data. An alternative would be to use segments from natural continuous speech extracted from multilingual corpora. This would provide more naturalistic input and broader availability of comparable data across languages. However, this would also make the test more distinct from the typical setups utilized in infant studies. Most centrally, the use of natural continuous speech would likely make the discrimination task more difficult due to added contextual variability, which would make comparison against infant effect size(s) again more problematic since infant data comes from relatively simplistic stimuli.

As for the generality of our present findings, the limitations of Experiment \#1 in terms of model training data apply, namely that we are not using realistic infant input data in terms of quality or quantity in order to properly simulated language experiences in the included infant age range. Another factor to keep in mind is the funnel plot asymmetry observed in the sample data from infant studies (Figs. 10a and 10b), which is as a potential indication of publication bias in the literature regarding vowel discrimination (see also Tsuji and Cristia, 2014). If true, this would mean that the actual effect size for infants could be lower than the estimated one, or even a null effect in the worst case (Lipsey and Wilson, 2001). In addition, the number of studies used to estimate the effect size for the non-native vowel contrasts is quite limited, which increases the uncertainty in the related overall effect size as well as in the estimate of developmental trajectory. Compare now the approaches followed across the two experiments: In Experiment \#1, we could rely on 49 effect sizes collected using essentially the same stimuli and procedure on 1066 infants, leading to lower heterogeneity; and the study was registered, leading to no publication bias; In contrast, the current Experiment \#2 has to face increased heterogeneity and potentially publication biases, in a dataset that is also smaller (non-native contrasts: 31 effect sizes, representing 641 infants). There is, however, a trade-off: Experiment \#1 only covered a small region of the potential space of stimuli, whereas Experiment \#2 could build on a larger set of stimuli, including a variety of phonological contrasts. These considerations are important for our Guideline \#4 on representativeness. Limitations on number of studies included in the meta-analysis and interpretation of those results for reference data also highlights the need for evolving evaluation practices that can be updated with new empirical data.

Together, the considerations related to training data limitations and baby data representativeness lead us to avoid making any strong conclusions regarding our models' compatibility with behavioral data. The strength of the current work is, as with the first experiment, to showcase the basic elements of the meta-analytic evaluation process.

\subsection{Are APC and CPC models compatible with human data overall?}

One overall concern, as mentioned in the Introduction, is overfitting. The risk of overfitting decreases as more language capabilities and moderator values are considered in parallel in a meta-analytic model evaluation approach. We can then ask, taking into account Experiments \# 1 and 2, are both models compatible with the infant data for overall effect sizes and changes with experience?

When looking at the models after $100 \mathrm{~h}$ of training data, and focusing on overall effect sizes, the CPC model was compatible with infant data for both IDS preference and vowel discrimination capabilities. In contrast, the APC model only yielded an effect size compatible for the vowel discrimination capability, because its effect size for IDS preference was too small. This illustrates that looking at only one language capability at a time and focusing only on the main effect provides relatively limited information on the model compatibility with the human data.

This does not mean that CPC provided a perfect fit, as it failed in the phase when developmental trajectories, i.e., effect sizes as a function of the amount of input data, were inspected. Specifically, 
the CPC model has a developmental trajectory for vowel discrimination (native and non-native contrasts) that are incongruous with those described by meta-analytic data; and, in the case of nonnative vowel discrimination, it is incongruous also with dominant theories of perceptual attunement (e.g., Kuhl et al., 2008) as the model also improves on non-native vowels with experience. Although these results are difficult to interpret and will surprise many readers, they suffice to make our key points, which is that the proposed meta-analytic model already provides considerable constraints for assessing whether a given model fits extant infant data. For a more systematic evaluation, we should take into account that the age range of infant studies is much more diverse than our present binary untrained/somewhat trained distinction used for the models. To better simulate infant learning, more systematic computational experiments with a broader range of amount of training data would be required, ideally matching with the scale of speech input that infants get during their first year of life.

\section{General discussion}

This paper introduced a basic framework to evaluate computational models of early language acquisition against robust human reference, as obtained from meta-analyses on different language capabilities. We established a connection between the meta-analysis outcomes (in terms of effect sizes) and behavior of computational models, and explored the extent to which that comparison between human and model effects is possible. In order to establish this connection, we defined two standard output requirements that each model must satisfy (or can be augmented with) in order to enable comparison with a range of empirical infant studies, namely internal latent representations and attentional preference scores. We also reviewed some basic considerations in creating computational tests for language capabilities such that the tests are both representative of the phenomenon of interest and flexibly applicable to various types of computational models. We tested the evaluation protocol implementation with two predictive coding models of speech representation learning, which were evaluated against state-of-the-art knowledge on infant IDS preference (Experiment \#1; exemplifying the use of attentional preference scores, and data from a large-scale collaboration) and vowel discrimination (Experiment \#2; demonstrating the use of latent representations, and data from MetaLab). Our experiments showed that the proposed evaluation framework facilitates examination of models even when the capabilities and associated behavioral methodologies vary between experiments.

However, meta-analytic evaluation of computational models underlying language acquisition is still challenging in many respects. Despite our present efforts, precise quantitative comparison between behavioral measures obtained from infants and outputs obtained from models is still complicated. In this paper, benchmarking is not done statistically; specifically, we have not provided tests of statistical difference or equivalence of developmental patterns, against current statistical recommendations (Lakens, 2017), including for computational modeling of cognition (Palminteri et al., 2017). The main reason for this departure is that measurement of infants' language behaviours is noisy, and the amount of noise is difficult, if not impossible, to estimate precisely. This means that empirical research, whether individual studies or meta-analyses across them, underestimate the magnitude of the real effect, and it is difficult to derive an upper bound for the underlying "true" effect. In addition, the current standard meta-analytic practice does not provide information on individual variation across infants, but only an estimate of the mean effect size in the population. This means that, when testing only one model instance, there is no systematic way to test whether the model could be representative of any individual infant in the population. Instead, a comparison of model and infant mean effects can only be carried out, where the model with an effect size equal 
or larger than that of infants can be considered as compatible with the human data.

Another challenge is that targets for modeling are constantly evolving, as empirical techniques for assessing infants' skills improve. In addition, even when focusing on a task like native vowel discrimination, replication and extension studies provide new data for phenomena that were postulated based on a limited number of studies with limited sample sizes; for instance, current data in MetaLab do not reveal improvements in native vowel discrimination with infant age, unlike conclusions drawn from previously-available infant data (Tsuji and Cristia, 2014). From the modeling perspective, this means that not only the models undergo continuous development, but also that the empirical reference point for the models changes with improving understanding of child language development. Hence, an effective continuous cycle of improved models and improved evaluation practices is desirable to maintain a strong coupling between the behavioral and computational research. We have argued that this could be addressed using cumulative resources such as MetaLab and standard meta-analytic procedures as integral components of model evaluation protocols.

We also touched the issue of specialized task-dependent computational models and models that could explain several language phenomena with a limited set of learning mechanisms. Ideally, a parsimonious model of child language development is expected to simulate different language capabilities in terms of their developmental trajectories across an age range relevant to the phenomena of interest. In the present form, the meta-analytic approach to evaluation does not provide particularly fine-grained targets for models to reach if only one language capability is in the focus (due to the lack of upper bounds for effect sizes discussed above). However, when multiple language capabilities are considered in parallel, and when each of those is being studied as a function of different moderators (especially infant age), the meta-analytic approach starts to provide tighter constraints on the space of possible models that are still compatible with the infant data. Hence, we believe that multi-capability testing accompanied by meta-analytic tools is particularly suitable for working towards more integrated models of learning that could actually follow infant developmental trajectories across a range of language-related phenomena, i.e., models that exhibit changes in their behavior as they experience more input data in a similar manner that infant behavior changes with development. Notice that modeling and evaluation of developmental trajectories is much more limited with data from individual empirical studies, as they usually have limited subject age distributions for practical reasons, and even more difficult if evaluation focuses on comparing models against linguistic theory.

Overall, the benefits of using meta-analysis at the heart of the testing scheme go beyond mean effects, with analyses that allow investigation of more complex relationships between variables that may interact during language development. This parallels the recent developments in empirical language development research, where approaches towards more comprehensive theories that make systematic contact with large-scale data have started to emerge (Cristia et al., 2021; Karcheris et al., 2021; Lewis et al., 2016), whereas the previous influential theories have been largely descriptive in nature (e.g., Jusczyk, 1993; Kuhl et al., 2008; Werker and Curtin, 2005).

\subsection{Limitations and open issues}

This work has laid down the basic framework for model evaluation, but these should be viewed only as the very initial steps towards systematic model verification with robust empirical data. In practice, the present approach has several limitations and challenges.

A cornerstone for evaluation on the machine side is the assumption of representativeness of the computational test administered to a model. This is not only a theoretical assumption required to make behavior comparison in two worlds (human and machine) feasible, but also a key issue in psychological theory and practice. Indeed, there is a whole field of psychology (psychometrics) that attempts to develop tasks that have high external validity, meaning that they generalize to new 
populations and capture well the construct or phenomenon they are intended to capture. It is beyond the scope of the present work to assess whether the infant tasks included here (infant-directed speech, vowel discrimination) and others available today from large-scale collaborations across babylabs and meta-analyses indeed have high external validity. ${ }^{16}$ In addition, it behooves the creators of such datasets to consider whether they have fully represented the scope of a capability in a representative manner, or whether meta-analytic results reflect unequal distributions of included studies (varying in e.g. design and stimuli). Instead, we have recommended to implement original behavioral tests for their computational counterparts, to better ensure equality in representativeness. While comparison between model and human behavior is facilitated by accurately following the designs of the empirical studies, any limitations in the empirical study designs will also carry over to the computational side. In addition, many of the empirical study design choices may originate from the difficulties in testing infant subjects, balancing between representativeness and feasibility of conducting the desired measurements. The latter is generally not an issue with computational studies, where a single model can be subjected to practically as many test trials as one wishes. Therefore, it may sometimes make sense to diverge from an empirical study design in certain (informed) ways when designing a computational test for the language phenomenon of interest. However, there is no unanimous way to balance out representativeness, accuracy, and comparability of the test with human data. In order to have at least some handle on this issue, our suggestion is to design tests with transparent moderator information of included trials, such as clearly separating empirical replication trials from those that extend the test beyond the behavioral literature. With the help of moderators, a more systematic investigation can be conducted on i) whether the model fits to human data that exists, when measured with similar test tokens as humans, and, ii) whether other reasonable ways of testing the same model on the same capability lead to compatible or diverging findings, hence informing the researcher also on the representativeness of the empirical practices used to test infants on the same capability.

Another issue not covered by the present work is the question of individual variation in humans and models (see Haines et al., 2020 for recent arguments about the importance of such a perspective). In Section 3, we drew a parallel between an individual model instance (initialized with certain parameters and trained with certain language experiences) and an individual human participant. Then, we ended up comparing population-level meta-analytic effect sizes to the effects measured from an individual model. This comes with the implicit assumption that the tested model should reflect an average $^{17}$ test subject across all the tested capabilities in order to be considered as accurate. Given the analogy, what we would ideally like to know is whether the tested model is a plausible substitute for a human individual that would be subjected to the same set of tests, i.e., likely performing above population average in some tests and below in others. However, the present meta-analytic approaches, as conducted on summary statistics from individual studies, do not provide estimates on the range of effects observed across individuals. This prevents statistical testing of whether a data point (model effect size) can reasonably originate from the distribution of human effects. Instead, the procedure only provides estimate of the mean together with its confidence intervals. Even though Individual Participant Data (IPD) meta-analyses could be an alternative, they are more laborious to carry out and less mature than meta-analyses based on aggregates (Wang et al., 2021). Given only access to population level statistics on the human side, the comparable thing would be to test a pool of models sampled from a model population (see Section 3.1) and compute comparable statistics for them before human-machine comparison. In principle, the basic formalism defined in

\footnotetext{
${ }^{16}$ It would be of high importance to do this, as argued by Grahek et al. (2021). Unfortunately, there is some evidence that the current sampling of the world's infant population is far from representative (Kidd and Garcia, 2021; Singh et al., 2021), which raises concerns for generalizability, as do low test-retest reliability reports (Cristia et al., 2016).

${ }^{17}$ Or above average, considering the difficulty in defining upper bound for the human effect sizes (see Section 3.4).
} 
Section 3.1 provides a means to derive more systematic comparison of testing multiple participants across multiple experimental studies, and similarly testing multiple model variants across multiple tests. However, this is currently often computationally intractable with complex large-scale models, especially if realistic-scale training data is to be used in the training process. In addition, the concept of individual model instances (with their specific initial and learned parameters) being members of some more general definition of (super-level) "a model" (in analogy to behavioral test participants being instances of "human") is not something that exists in the standard conceptual toolkit of computational modeling (or machine learning). Moreover, infant data is so noisy it is at present unclear whether individual performance can be measured reliably (Cristia et al., 2016), to the point that some investigations suggest it is equivalent to collect more trials from fewer infants or vice versa (see DeBolt et al., 2020 for a recent discussion). Therefore, a systematic treatment of how to evaluate collectives of models instead of individual model instances, and what would be the implication of such modeling practices, is beyond the scope of the present paper and is thereby left for follow-up work.

It is also good to remember that meta-analysis is no magic bullet. Meta-analyses are as good as the data that goes into them, and the statistical modeling performed. This means that metaanalytic findings can depend on analysis practices (e.g., study inclusion criteria or statistical model used in the analysis), and the results can also change with new empirical data. In addition, the effect sizes from meta-analyses can provide a biased estimation if the underlying literature is biased, although meta-analytic practices come with a means to identify the presence of publication bias in the literature (Egger et al., 1997). This calls for transparent, open, and standardized meta-analytic practices (see Polanin et al., 2020; Tsuji et al., 2014 for more details). Since improving meta-analytic practices is a field of expertise on its own, the practical approach for most computational modeling researchers is to simply adapt already published expert-based meta-analyses as long as they exist for the capability of interest. Those who have the expertise (or collaborations) to conduct their own custom meta-analyses for the question at hand can then do so.

Finally, the mathematical basis for comparing models and humans described in Section 3 was not fully utilized in this first study. Given the finite scope of this paper, we have not made comprehensive use of the analogy between model instances and human participants, or pools of model instances as analogs of a larger human population. Nor did we discuss how parametrization of models could be related to individual developmental processes, "innate" priors", or characteristics shared by a larger population of models. However, we find it relevant to lay down one formal basis for approaching these questions in a principled manner, providing a starting point for future work that could address the shortcomings discussed above - a concrete starting point to be revised and replaced as needed.

\section{Conclusions}

We have introduced the concept of evaluating computational models of child language development against empirical human data aggregated from multiple studies. Motivation for this stems from the need to circumvent the issues in replicability and representativeness of individual limited-sample empirical studies on infants, and to thereby derive more robust and reliable reference against which computational models could be compared to. Following this goal, we have provided a number of practical suggestions and general guidelines as a starting point for meta-analytic model evaluation, but we have also brought up several issues and limitations in comparing computational models with infant data. Some of these are simply a matter of requiring more manual work in implementing suitable tests and simulation experiments to conduct proper comprehensive tests for the models of interest. Some others are more fundamental in nature (e.g., deriving upper bounds for model effect 
sizes), and will require new ways of thinking about model evaluation. In general, there is plenty of room for improving the statistical approaches for quantifying variability in human and model data, and in conducting the comparisons between two. These considerations also demonstrate how the conceptually simple idea of comparing infant effects to model effects turns into a large set of relatively complicated considerations of how to test the models in a manner that enables comparison of model performance with that of humans. Nevertheless, we also feel that cumulative science practices and resources together with a multidisciplinary approach to the study of language development are a solid basis for handling the challenges posed by this type of evaluation.

We would also like to emphasize the complementarity of the proposed evaluation practices with respect to other alternatives. The present meta-analytic approach is a means to obtain high-level holistic view on a model across one or more language capabilities, checking against the most robust patterns available in the behavioral data. It does not exclude or replace the need for model fitting to data from individual behavioral experiments to study more fine-grained phenomena and details in the underlying cognitive mechanisms, at least as long as there are not sufficiently many replications of the detailed experimental manipulations to support meta-analytic fusion (see Cristia et al., 2021 for further discussion on the place of meta-analyses versus single studies in testing a theory's explanatory adequacy). In addition, the meta-analytic approach does not replace the need and motivation for comparing model behavior against linguistic ground truth on the processed language inputs - a correlate of adult-like language capability and how science views language as a structured system (Section 2)-but complements it by checking for model compatibility with infant data and providing a way to model different stages of language development for different language capabilities. By simultaneously ensuring model compatibility with infant data while probing for linguistic representations in the models, a better understanding of the language learning in infancy and language as a phenomenon in general can be developed.

In general, we would like to subject our present guidelines and ideas for further development in the language research community, and also to encourage the community to participate to further development of computational tests for language capabilities to compare against infants in similar settings.

\section{Acknowledgements}

Authors MACB and OR were supported by Academy of Finland grants no. 314602, 320053, and 345365. AC was funded by Agence Nationale de la Recherche (ANR-16-DATA-0004 ACLEW, ANR17-EURE-0017), the ERC (ExELang, 101001095), and a J. S. McDonnell Foundation Understanding Human Cognition Scholar Award.

The code to reproduce reported experiments is available at https://github.com/SPEECHCOG/ metaeval_experiments/. The Isolated Vowel Corpus is available at https://github.com/SPEECHCOG/ isolated_vowels_corpus/.

\section{References}

Alishahi, A. (2010). Computational modeling of human language acquisition. Morgan \& Claypool Publishers. https://doi.org/10.2200/S00304ED1V01Y201010HLT011

Alishahi, A., Barking, M., \& Chrupała, G. (2017). Encoding of phonology in a recurrent neural model of grounded speech. Proceedings of the 21st Conference on Computational Natural Language Learning (CoNLL 2017), pp. 368-378. 
Alishahi, A., Chrupala, G., Cristià, A., Dupoux, E., Higy, B., Lavechin, M., Räsänen, O., \& Yu, C. (2021). ZR-2021VG: Zero-Resource Speech Challenge, Visually-Grounded Language Modelling Track, 2021 edition. CoRR, abs/2107.06546. https://arxiv.org/abs/2107.06546

Baevski, A., Zhou, H., Mohamed, A., \& Auli, M. (2020). wav2vec 2.0: A Framework for SelfSupervised Learning of Speech Representations, 1-19. http://arxiv.org/abs/2006.11477

Belinkov, Y., \& Glass, J. R. (2018). Analysis methods in neural language processing: A survey. CoRR, abs/1812.08951. http://arxiv.org/abs/1812.08951

Bergmann, C., Tsuji, S., Piccinini, P. E., Lewis, M. L., Braginsky, M., Frank, M. C., \& Cristia, A. (2018). Promoting Replicability in Developmental Research Through Meta-analyses: Insights From Language Acquisition Research. Child Development, 89(6), 1996-2009. https: //doi.org/10.1111/cdev.13079

Bhati, S., Villalba, J., Żelasko, P., Moro-Velazquez, L., \& Dehak, N. (2021). Segmental Contrastive Predictive Coding for Unsupervised Word Segmentation. Proceedings of the Annual Conference of the International Speech Communication Association (Interspeech), pp. 366-370. https://doi.org/10.21437/Interspeech.2021-1874

Bishop, C. M. (2006). Pattern recognition and machine learning. Springer.

Bohn, O.-S., \& Polka, L. (2001). Target spectral, dynamic spectral, and duration cues in infant perception of German vowels. The Journal of the Acoustical Society of America, 110(1), 504-515. https://doi.org/10.1121/1.1380415

Borsboom, D., van der Maas, H. L., Dalege, J., Kievit, R. A., \& Haig, B. D. (2021). Theory construction methodology: A practical framework for building theories in psychology. Perspectives on Psychological Science, 16, 756-766. https://doi.org/10.1177/1745691620969647

Brent, M. R. (1999). An efficient, probabilistically sound algorithm for segmentation and word discovery. Machine Learning, 34, 71-105. https://doi.org/10.1023/A:1007541817488

Cheour, M., Alho, K., Čeponiené, R., Reinikainen, K., Sainio, K., Pohjavuori, M., Aaltonen, O., \& Näätänen, R. (1998). Maturation of mismatch negativity in infants. International Journal of Psychophysiology, 29(2), 217-226. https://doi.org/10.1016/S0167-8760(98)00017-8

Chomsky, N. (1965). Aspects of the theory of syntax. The MIT Press.

Chorowski, J., Weiss, R. J., Bengio, S., \& van den Oord, A. (2019). Unsupervised speech representation learning using wavenet autoencoders. IEEE/ACM Transactions on Audio, Speech, and Language Processing, 27(12), 2041-2053. https://doi.org/10.1109/TASLP.2019.2938863

Chrupala, G. (2021). Visually grounded models of spoken language: A survey of datasets, architectures and evaluation techniques. CoRR, abs/2104.13225. https://arxiv.org/abs/2104.13225

Chrupała, G., Gelderloos, L., \& Alishahi, A. (2017). Representations of language in a model of visually grounded speech signal. Proceedings of the 55th Annual Meeting of the Association for Computational Linguistics (Volume 1: Long Papers), pp. 613-622.

Chrupała, G., Higy, B., \& Alishahi, A. (2020). Analyzing analytical methods: The case of phonology in neural models of spoken language. Proc. 58th Annual Meeting of the Association for Computational Linguistics, pp. 4146-4156.

Chung, Y. A., \& Glass, J. (2019). Generative Pre-Training for Speech with Autoregressive Predictive Coding. ArXiv, abs/1910.1.

Chung, Y. A., Hsu, W. N., Tang, H., \& Glass, J. (2019). An unsupervised autoregressive model for speech representation learning. Proceedings of the Annual Conference of the International Speech Communication Association (Interspeech), pp. 146-150. https://doi.org/10.21437/ Interspeech.2019-1473

Coen, M. H. (2006). Self-supervised acquisition of vowels in American English. Proceedings of the 21st National Conference on Artificial Intelligence, 2, pp. 1451-1456. 
Cowan, N., Suomi, K., \& Morse, P. A. (1982). Echoic Storage in Infant Perception. Child Development, 53(4), 984-990.

Cristia, A., Seidl, A., Singh, L., \& Houston, D. (2016). Test-retest reliability in infant speech perception tasks. Infancy, 21(5), 648-667. https://doi.org/10.1111/infa.12127

Cristia, A., Tsuji, S., \& Bergmann, C. (2021). A meta-analytic approach to evaluating the explanatory adequacy of theories. https://doi.org/10.31219/osf.io/83kg2

Cruz Blandón, M. A., \& Räsänen, O. (2020). Analysis of predictive coding models for phonemic representation learning in small datasets. Workshop on Self-supervision in Audio and Speech at the 37th International Conference on Machine Learning.

De Seyne, S., Navarro, D. J., Collell, G., \& Perfors, A. (2021). Visual and affective multimodal models of word meaning in language and mind. Cognitive Science, 45, e12922. https://doi. org $/ 10.1111 / \operatorname{cogs} .12922$

de Boer, B., \& Kuhl, P. K. (2003). Investigating the role of infant-directed speech with a computer model. Acoustics Research Letters Online, 4, 129-134. https://doi.org/10.1121/1.1613311

DeBolt, M. C., Rhemtulla, M., \& Oakes, L. M. (2020). Robust data and power in infant research: A case study of the effect of number of infants and number of trials in visual preference procedures. Infancy, 25(4), 393-419. https://doi.org/10.1111/infa.12337

Dunbar, E., Algayres, R., Karadayi, J., Bernard, M., Benjumea, J., Cao, X. N., Miskic, L., Dugrain, C., Ondel, L., Black, A. W., Besacier, L., Sakti, S., \& Dupoux, E. (2019). The zero resource speech challenge 2019: TTS without T. Proceedings of the Annual Conference of the International Speech Communication Association (Interspeech), pp. 1088-1092. https: //doi.org/10.21437/Interspeech.2019-2904

Dunbar, E., Cao, X. N., Benjumea, J., Karadayi, J., Bernard, M., Besacier, L., Anguera, X., \& Dupoux, E. (2017). The zero resource speech challenge 2017. IEEE Automatic Speech Recognition and Understanding Workshop (ASRU), 323-330. https://doi.org/10.1109/ASRU. 2017.8268953

Dunst, C. J., Gorman, E., \& Hamby, D. W. (2012). Preference for infant-directed speech in preverbal young children. Center for Early Literacy Learning Reviews, 5(1), 1-13.

Dupoux, E. (2018). Cognitive science in the era of artificial intelligence: A roadmap for reverseengineering the infant language-learner. Cognition, 173, 43-59. https://doi.org/10.1016/j. cognition.2017.11.008

Dutoit, T., Pagel, V., Pierret, N., Bataille, E., \& van der Vrecken, O. (1996). The MBROLA project: Towards a set of high quality speech synthesizers free of use for non commercial purposes. Proceedings of the International Conference on Spoken Language Processing (ICSLP), 3, pp. 1393-1396. https://doi.org/10.1109/icslp.1996.607874

Egger, M., Smith, G. D., Schneider, M., \& Minder, C. (1997). Bias in meta-analysis detected by a simple, graphical test. British Medical Journal, 315(7109), 629-634. https://doi.org/10. 1136/bmj.315.7109.629

Elsner, M., Goldwater, S., \& Eisenstein, J. (2012). Bootstrapping a unified model of lexical and phonetic acquisition. Proceedings of the 50th Annual Meeting of the Association for Computational Linguistics, 184-193.

Fernald, A. (1985). Four-month-old infants prefer to listen to motherese. Infant Behavior and Development, 8(2), 181-195. https://doi.org/https://doi.org/10.1016/S0163-6383(85)80005-9

Fernald, A., Taeschner, T., Dunn, J., Papousek, M., de Boysson-Bardies, B., \& Fukui, I. (1989). A cross-language study of prosodic modifications in mothers' and fathers' speech to preverbal infants. Journal of Child Language, 16(3), 477-501. https://doi.org/10.1017/ S0305000900010679 
Fourtassi, A., \& Dupoux, E. (2014). A rudimentary lexicon and semantics help bootstrap phoneme acquisition. Proceedings of the Eighteenth Conference on Computational Natural Language Learning, pp. 191-200. https://doi.org/10.3115/v1/W14-1620

Frank, M. C., Alcock, K. J., Arias-Trejo, N., Aschersleben, G., Baldwin, D., Barbu, S., Bergelson, E., Bergmann, C., Black, A. K., Blything, R., Böhland, M. P., Bolitho, P., Borovsky, A., Brady, S. M., Braun, B., Brown, A., Byers-Heinlein, K., Campbell, L. E., Cashon, C., ... Soderstrom, M. (2020). Quantifying Sources of Variability in Infancy Research Using the Infant-Directed-Speech Preference. Advances in Methods and Practices in Psychological Science, 3(1), 24-52. https://doi.org/10.1177/2515245919900809

Frank, M. C., Bergelson, E., Bergmann, C., Cristia, A., Floccia, C., Gervain, J., Hamlin, J. K., Hannon, E. E., Kline, M., Levelt, C., et al. (2017a). A collaborative approach to infant research: Promoting reproducibility, best practices, and theory-building. Infancy, 22(4), 421-435. https://doi.org/10.1111/infa.12182

Frank, M. C., Bergelson, E., Bergmann, C., Cristia, A., Floccia, C., Gervain, J., Hamlin, J. K., Hannon, E. E., Kline, M., Levelt, C., Lew-Williams, C., Nazzi, T., Panneton, R., Rabagliati, H., Soderstrom, M., Sullivan, J., Waxman, S., \& Yurovsky, D. (2017b). A collaborative approach to infant research: Promoting reproducibility, best practices, and theory-building. Infancy, 22(4), 421-435. https://doi.org/10.1111/infa.12182

Frank, M. C., Braginsky, M., Yurovsky, D., \& Marchman, V. A. (2017). Wordbank: An open repository for developmental vocabulary data. Journal of Child Language, 44 (3), 677-694. https: //doi.org/10.1017/S0305000916000209

Frank, M. C., Goldwater, S., Griffiths, T. L., \& Tenenbaum, J. B. (2010). Modeling human performance in statistical word segmentation. Cognition, 117(2), 107-125. https://doi.org/10. 1016/j.cognition.2010.07.005

Frank, M. C., Goodman, N. D., \& Tenenbaum, J. (2009). Using speakers' referential intentions to model early cross-situational word learning. Psychological Science, 20, 578-585. https: //doi.org/10.1111/j.1467-9280.2009.02335.x

Fried, E. I. (2020). Theories and models: What they are, what they are for, and what they are about. Psychological Inquiry, 31(4), 336-344. https://doi.org/10.1080/1047840X.2020.1854011

Friston, K. (2005). A theory of cortical responses. Philosophical Transactions of the Royal Society of London. Series B, Biological Sciences, 360(1456), 815-836. https://doi.org/10.1098/rstb. 2005.1622

Friston, K. (2010). The free-energy principle: A unified brain theory? Nature Reviews Neuroscience, 11, 127-138. https://doi.org/10.1038/nrn2787

García-Martín, E., Rodrigues, C. F., Riley, G., \& Grahn, H. (2019). Estimation of energy consumption in machine learning. Journal of Parallel and Distributed Computing, 134, 75-88. https://doi.org/https://doi.org/10.1016/j.jpdc.2019.07.007

Goldwater, S., Griffiths, T. L., \& Johnson, M. (2009). A bayesian framework for word segmentation: Exploring the effects of context. Cognition, 112(1), 21-54. https://doi.org/10.1016/j. cognition.2009.03.008

Golinkoff, R. M., Can, D. D., Soderstrom, M., \& Hirsh-Pasek, K. (2015). (baby)talk to me: The social context of infant-directed speech and its effects on early language acquisition. Current Directions in Psychological Science, 24(5), 339-344. https://doi.org/10.1177/0963721415595345

Grahek, I., Schaller, M., \& Tackett, J. L. (2021). Anatomy of a psychological theory: Integrating construct-validation and computational-modeling methods to advance theorizing. Perspectives on Psychological Science, 16, 803-815. https://doi.org/10.1177/1745691620966794 
Guest, O., \& Martin, A. E. (2021). How computational modeling can force theory building in psychological science. Perspectives on Psychological Science, 16, 789-802. https://doi.org/10. $1177 / 1745691620970585$

Gutmann, M., \& Hyvärinen, A. (2010). Noise-contrastive estimation: A new estimation principle for unnormalized statistical models. Proceedings of the Thirteenth International Conference on Artificial Intelligence and Statistics, 9, pp. 297-304.

Haines, N., Kvam, P. D., Irving, L. H., Smith, C., Beauchaine, T. P., Pitt, M. A., Ahn, W.-Y., \& Turner, B. (2020). Learning from the reliability paradox: How theoretically informed generative models can advance the social, behavioral, and brain sciences. https://doi.org/ 10.31234/osf.io/xr7y3

Harwath, D., \& Glass, J. R. (2019). Towards visually grounded sub-word speech unit discovery. IEEE International Conference on Acoustics, Speech and Signal Processing (ICASSP 2019), pp. 3017-3021. https://doi.org/10.1109/ICASSP.2019.8682666

Harwath, D., Hsu, W.-N., \& Glass, J. R. (2019). Learning hierarchical discrete linguistic units from visually-grounded speech. CoRR, abs/1911.09602. http://arxiv.org/abs/1911.09602

Harwath, D., Recasens, A., Surıs, D., Chuang, G., Torralba, A., \& Glass, J. R. (2018). Jointly discovering visual objects and spoken words from raw sensory input. 15th European Conference on Computer Vision (ECCV 2018), 11210, pp. 659-677.

Harwath, D., Torralba, A., \& Glass, J. (2016). Unsupervised learning of spoken language with visual context. Advances in Neural Information Processing Systems 29: Annual Conference on Neural Information Processing Systems (NIPS 2016), pp. 1858-1866.

Havard, W. N., Chevrot, J. P., \& Besacier, L. (2019). Word recognition, competition, and activation in a model of visually grounded speech. Proceedings of the 23rd Conference on Computational Natural Language Learning (CoNLL), pp. 339-348. https://doi.org/10.18653/v1/k19-1032

Hedges, L. V., \& Olkin, I. (1985). Statistical methods for meta-analysis. Academic Press. https: //doi.org/10.1016/B978-0-08-057065-5.50005-1

Hillenbrand, J., Getty, L. A., Clark, M. J., \& Wheeler, K. (1995). Acoustic characteristics of American English vowels. Acoustic characteristics of American English vowels, 97(5), 3099-3111. https://doi.org/10.1121/1.411872

Howard, I. S., \& Messum, P. (2011). Modeling the development of pronunciation in infant speech acquisition. Motor Control, 15(1), 85-117. https://doi.org/0.1123/mcj.15.1.85

Hunter, M. A., \& Ames, E. W. (1988). A multifactor model of infant preferences for novel and familiar stimuli. Advances in Infancy Research, 5, 69-95.

Ioannidis, J. (2005). Why most published research findings are false. PLoS Medicine, 2(8), e124. https://doi.org/10.1126/science.aac4716

Jusczyk, P. W. (1993). From general to language-specific capacities: the WRAPSA Model of how speech perception develops. Journal of Phonetics, 21 (1-2), 3-28. https://doi.org/10.1016/ s0095-4470(19)31319-1

Kachergis, G., Yu, C., \& Shiffrin, R. M. (2017). A bootstrapping model of frequency and context effects in word learning. Cognitive Science, 41(3), 590-622. https://doi.org/10.1111/cogs. 12353

Kager, R., Pater, J., \& Zonneveld, W. (2004). Constraints in Phonological Acquisition. Cambridge University Press. https://doi.org/10.1017/CBO9780511486418

Kamper, H., Jansen, A., \& Goldwater, S. (2016). Unsupervised word segmentation and lexicon discovery using acoustic word embeddings. IEEE/ACM Transactions on Audio Speech and Language Processing, 24(4), 669-679. https://doi.org/10.1109/TASLP.2016.2517567

Kamper, H., \& van Niekerk, B. (2021). Towards Unsupervised Phone and Word Segmentation Using Self-Supervised Vector-Quantized Neural Networks. Proceedings of the Annual Conference 
of the International Speech Communication Association (Interspeech), pp. 1539-1543. https: //doi.org/10.21437/Interspeech.2021-50

Karcheris, G., Marchman, V. A., \& Frank, M. C. (2021). Towards a "standard model" of early language learning. PsyArXiv preprints. https://doi.org/10.31234/osf.io/yhrb4

Kharitonov, E., Lee, A., Polyak, A., Adi, Y., Copet, J., Lakhotia, K., Nguyen, T.-A., Rivière, M., Mohamed, A., Dupoux, E., \& Hsu, W.-N. (2021). Text-free prosody-aware generative spoken language modeling. https://arxiv.org/abs/2109.03264

Khorrami, K., \& Räsänen, O. (2021). Can phones, syllables, and words emerge as side-products of cross-situational audiovisual learning? - A computational investigation. Language Development Research. https://doi.org/10.34842/w3vw-s845

Kidd, C., Piantadosi, S. T., \& Aslin, R. N. (2012). The goldilocks effect: Human infants allocate attention to visual sequences that are neither too simple nor too complex. PLOS One. https://doi.org/10.1371/journal.pone.0036399

Kidd, E., \& Garcia, R. (2021). How diverse is child language acquisition? https://doi.org/10.31234/ osf.io/jpeyq

Kirby, S. (2001). Spontaneous evolution of linguistic structure-an iterated learning model of the emergence of regularity and irregularity. IEEE Transactions on Evolutionary Computation, 5(2), 102-110. https://doi.org/10.1109/4235.918430

Kreuk, F., Keshet, J., \& Adi, Y. (2020). Self-supervised contrastive learning for unsupervised phoneme segmentation. Proceedings of the Annual Conference of the International Speech Communication Association (Interspeech), pp. 3700-3704. https:// doi.org/10.21437/ Interspeech.2020-2398

Kuhl, P. K. (1979). Speech perception in early infancy: Perceptual constancy for spectrally dissimilar vowel categories. Journal of the Acoustical Society of America, 66(6), 1668-1679. https: //doi.org/10.1121/1.383639

Kuhl, P. K., Conboy, B. T., Coffey-Corina, S., Padden, D., Rivera-Gaxiola, M., \& Nelson, T. (2008). Phonetic learning as a pathway to language: New data and native language magnet theory expanded (nlm-e). Philosophical Transactions of the Royal Society B: Biological Sciences, 363, 979-1000. https://doi.org/10.1098/rstb.2007.2154

Kuhl, P. K., \& Miller, J. D. (1982). Discrimination of auditory target dimensions in the presence or absence of variation in a second dimension by infants. Perception 85 Psychophysics, 31(3), 279-292. https://doi.org/10.3758/BF03202536

Lake, B. M., \& Murphy, G. L. (2021). Word meaning in minds and machines. Psychological Review, online pre-print. https://doi.org/10.1037/rev0000297

Lakens, D. (2017). Equivalence tests: A practical primer for t tests, correlations, and meta-analyses. Social Psychological and Personality Science, 8(4), 355-362. https://doi.org/10.1177/ 1948550617697177

Lakens, D. (2013). Calculating and reporting effect sizes to facilitate cumulative science: a practical primer for t-tests and ANOVAs. Frontiers in Psychology, 4, article 863. https://doi.org/10. 3389/fpsyg.2013.00863

Landauer, T. K., \& Dumais, S. T. (1997). A solution to plato's problem: The latent semantic analysis theory of acquisition, induction, and representation of knowledge. Psychological Review, 104, 211-240.

Larsen, E., Cristia, A., \& Dupoux, E. (2017). Relating unsupervised word segmentation to reported vocabulary acquisition. Proceedings of the Annual Conference of the International Speech Communication Association (Interspeech), 2198-2202. 
Lewis, M., Braginsky, M., Tsuji, S., Bergmann, C., Piccinini, P., Cristia, A., \& Frank, M. C. (2016). A quantitative synthesis of early language acquisition using meta-analysis. PsyArXiv preprints. https://doi.org/10.31234/osf.io/htsjm

Lipsey, M. W., \& Wilson, D. B. (2001). Practical meta-analysis. Sage Publications, Inc.

Magnuson, J. S., You, H., Luthra, S., Li, M., Nam, H., Escabí, M., Brown, K., Allopenna, P. D., Theodore, R. M., Monto, N., \& Rueckl, J. G. (2020). EARSHOT: A Minimal Neural Network Model of Incremental Human Speech Recognition. Cognitive Science, 44(4), e12823. https: //doi.org/10.1111/cogs.12823

Marean, G. C., Werner, L. A., \& Kuhl, P. K. (1992). Vowel Categorization by Very Young Infants. Developmental Psychology, 28(3), 396-405. https://doi.org/10.1037/0012-1649.28.3.396

Mazuka, R., Hasegawa, M., \& Tsuji, S. (2014). Development of non-native vowel discrimination: Improvement without exposure. Developmental Psychobiology, 56(2), 192-209. https://doi. org/10.1002/dev.21193

Merkx, D., Frank, S. L., \& Ernestus, M. (2019). Language Learning Using Speech to Image Retrieval. Proceedings of the Annual Conference of the International Speech Communication Association (Interspeech), pp. 1841-1845. https://doi.org/10.21437/Interspeech.2019-3067

Meyer, B. T., Jürgens, T., Wesker, T., Brand, T., \& Kollmeier, B. (2010). Human phoneme recognition depending on speech-intrinsic variability. The Journal of the Acoustical Society of America, 128(5), 3126-3141. https://doi.org/10.1121/1.3493450

Michel, P., Räsänen, O., Thiollière, R., \& Dupoux, E. (2016). Improving phoneme segmentation with recurrent neural networks. CoRR, abs/1608.00508. http://arxiv.org/abs/1608.00508

Miller, J. L., \& Eimas, P. D. (1979). Organization in infant speech perception. Canadian Journal of Psychology/Revue canadienne de psychologie, 33(4), 353-367. https://doi.org/10.1037/ h0081732

Minagawa-Kawai, Y., Mori, K., Naoi, N., \& Kojima, S. (2007). Neural attunement processes in infants during the acquisition of a language-specific phonemic contrast. Journal of Neuroscience, 27(2), 315-321. https://doi.org/10.1523/JNEUROSCI.1984-06.2007

Moulin-Frier, C., Diard, J., Schwartz, J.-L., \& Bessière, P. (2015). Cosmo ("communicating about objects using sensory-motor operations"): A bayesian modeling framework for studying speech communication and the emergence of phonological systems [On the cognitive nature of speech sound systems]. Journal of Phonetics, 53, 5-41. https://doi.org/10.1016/j.wocn. 2015.06.001

Mugitani, R., Pons, F., Fais, L., Dietrich, C., Werker, J. F., \& Amano, S. (2009). Perception of Vowel Length by Japanese- and English-Learning Infants. Developmental Psychology, 45(1), 236247. https://doi.org/10.1037/a0014043

Mulak, K. E., Bonn, C. D., Chládková, K., Aslin, R. N., \& Escudero, P. (2017). Indexical and linguistic processing by 12-month-olds: Discrimination of speaker, accent and vowel differences. PLoS ONE, 12(5), 1-19. https://doi.org/10.1371/journal.pone.0176762

Nguyen, T. A., de Seyssel, M., Rozé, P., Rivière, M., Kharitonov, E., Baevski, A., Dunbar, E., \& Dupoux, E. (2020). The Zero Resource Speech Benchmark 2021: Metrics and baselines for unsupervised spoken language modeling. http://arxiv.org/abs/2011.11588

Nikolaus, M., \& Fourtassi, A. (2021). Evaluating the acquisition of semantic knowledge from crosssituational learning in artificial. In Proceedings of the 12h Workshop on Cognitive Modeling and Computational Linguistics, pp. 200-210.

Nixon, J. S. (2020). Of mice and men: Speech sound acquisition as discriminative learning from prediction error, not just statistical tracking. Cognition, 197, e104081. https://doi.org/10. 1016/j.cognition.2019.104081 
Oakes, L. M. (2017). Sample size, statistical power, and false conclusions in infant looking-time research. Infancy, 22(4), 436-469. https://doi.org/10.1111/infa.12186

Oord, A. v. d., Li, Y., \& Vinyals, O. (2018). Representation Learning with Contrastive Predictive Coding. CoRR, abs/1807.0. https://arxiv.org/abs/1807.03748

Open Science Collaboration. (2015). Estimating the reproducibility of psychological science. Science, $349(6251)$. https://doi.org/10.1126/science.aac4716

Oudeyer, P.-Y. (2005). The self-organization of speech sounds. Journal of Theoretical Biology, 233, 435-449. https://doi.org/10.1016/j.jtbi.2004.10.025

Oudeyer, P.-Y., Kachergis, G., \& Schueller, W. (2019). Computational and robotic models of early language development: a review. International Handbook of Language Acquisition (pp. 76101). Routledge. https://doi.org/10.4324/9781315110622-5

Palminteri, S., Wyart, V., \& Koechlin, E. (2017). The importance of falsification in computational cognitive modeling. Trends in Cognitive Sciences, 21(6), 425-433. https://doi.org/10.1016/ j.tics.2017.03.011

Panayotov, V., Chen, G., Povey, D., \& Khudanpur, S. (2015). Librispeech: An ASR corpus based on public domain audio books. 2015 IEEE International Conference on Acoustics, Speech and Signal Processing (ICASSP), 5206-5210. https://doi.org/10.1109/ICASSP.2015.7178964

Park, A. S., \& Glass, J. R. (2008). Unsupervised pattern discovery in speech. IEEE Transactions on Audio, Speech, and Language Processing, 16(1), 186-197. https://doi.org/10.1109/TASL. 2007.909282

Peterson, D. (2016). The baby factory: Difficult research objects, disciplinary standards, and the production of statistical significance. Socius, 2, 1-10. https://doi.org/10.1177/2378023115625071

Pierrehumbert, J. B. (2003). Phonetic Diversity, Statistical Learning, and Acquisition of Phonology. Language and Speech, 46(2-3), 115-154. https://doi.org/10.1177/00238309030460020501

Polanin, J. R., Hennessy, E. A., \& Tsuji, S. (2020). Transparency and reproducibility of metaanalyses in psychology: A meta-review. Perspectives on Psychological Science, 15(4), 10261041. https://doi.org/10.1177/1745691620906416

Räsänen, O. (2012). Computational modeling of phonetic and lexical learning in early language acquisition: Existing models and future directions. Speech Communication, 54(9), 975-997. https://doi.org/10.1016/j.specom.2012.05.001

Räsänen, O. (2014). Basic cuts revisited: Temporal segmentation of speech into phone-like units with statistical learning at a pre-linguistic level. Proceedings of the 36th Annual Meeting of the Cognitive Science Society, pp. 2817-2822.

Räsänen, O., Doyle, G., \& Frank, M. C. (2015). Unsupervised word discovery from speech using automatic segmentation into syllable-like units. Proceedings of the Annual Conference of the International Speech Communication Association (Interspeech), 3204-3208. https://doi. org/10.21437/Interspeech.2015-645

Räsänen, O., Doyle, G., \& Frank, M. C. (2018). Pre-linguistic segmentation of speech into syllablelike units. Cognition, 171, 130-150. https://doi.org/10.1016/j.cognition.2017.11.003

Räsänen, O., \& Rasilo, H. (2015). A joint model of word segmentation and meaning acquisition through cross-situational learning. Psychological Review, 122(4), 792-829. https://doi.org/ $10.1037 / \mathrm{a} 0039702$

Räsänen, O., Seshadri, S., Lavechin, M., Cristia, A., \& Casillas, M. (2021). ALICE: An open-source tool for automatic measurement of phoneme, syllable, and word counts from child-centered daylong recordings. Behavior Research Methods, 53, 818-835. https://doi.org/doi.org/10. 3758

Rasilo, H., \& Räsänen, O. (2017). An online model for vowel imitation learning. Speech Communication, 86, 1-23. https://doi.org/10.1016/j.specom.2016.10.010 
Ribeiro, M. T., Wu, T., Guestrin, C., \& Singh, S. (2020). Beyond Accuracy: Behavioral Testing of NLP models with CheckList, 4902-4912. http://arxiv.org/abs/2005.04118

Robinaugh, D. J., Haslbeck, J. M., Ryan, O., Fried, E. I., \& Waldorp, L. J. (2021). Invisible hands and fine calipers: A call to use formal theory as a toolkit for theory construction. Perspectives on Psychological Science, 16(4), 725-743.

Rouditchenko, A., Boggust, A., Harwath, D., Chen, B., Joshi, D., Thomas, S., Audhkhasi, K., Kuehne, H., Panda, R., Feris, R., Kingsbury, B., Picheny, M., Torralba, A., \& Glass, J. (2021). AVLnet: Learning Audio-Visual Language Representations from Instructional Videos. Proceedings of the Annual Conference of the International Speech Communication Association (Interspeech), 1584-1588. https://doi.org/10.21437/Interspeech.2021-1312

Roy, D. (2005). Grounding words in perception and action: Computational insights. Trends in Cognitive Sciences, 9(8), 389-396. https://doi.org/10.1016/j.tics.2005.06.013

Sakoe, H., \& Chiba, S. (1978). Dynamic Programming Algorithm Optimization for Spoken Word Recognition. IEEE Transactions on Acoustics, Speech, and Signal Processing, 26(1), 43-49. https://doi.org/10.1109/TASSP.1978.1163055

Salvi, G., Montesano, L., Bernardino, A., \& Santos-Victor, J. (2012). Language bootstrapping: Learning word meanings from perception-action association. IEEE Transactions on Systems, Man, and Cybernetics, Part B (Cybernetics), 42(3), 660-671. https://doi.org/10. 1109/TSMCB.2011.2172420

Scharenborg, O., Ernestus, M., \& Wan, V. (2007). Segmentation of speech: child's play? Proceedings of the Annual Conference of the International Speech Communication Association (Interspeech), pp. 1953-1956. https://doi.org/10.21437/Interspeech.2007-47

Schatz, T., Feldman, N. H., Goldwater, S., Cao, X. N., \& Dupoux, E. (2021). Early phonetic learning without phonetic categories: Insights from large-scale simulations with realistic input. Proceedings of the National Academy of Sciences of the United States of America, 118(7), e2001844118. https://doi.org/10.1073/pnas.2001844118

Schatz, T., Peddinti, V., Bach, F., Jansen, A., Hermansky, H., \& Dupoux, E. (2013). Evaluating speech features with the minimal-pair ABX task: Analysis of the classical MFC/PLP pipeline. Proceedings of the Annual Conference of the International Speech Communication Association (Interspeech), pp. 1781-1785.

Schatz, T., Peddinti, V., Cao, X. N., Bach, F., Hermansky, H., \& Dupoux, E. (2014). Evaluating speech features with the Minimal-Pair ABX task (II): Resistance to noise. Proceedings of the Annual Conference of the International Speech Communication Association (Interspeech), pp. 915-919.

Scholten, S., Merkx, D., \& Scharenborg, O. (2021). Learning to recognise words using visually grounded speech. 2021 IEEE International Symposium on Circuits and Systems (ISCAS), pp. 1-5. https://doi.org/10.1109/ISCAS51556.2021.9401692

Shain, C., \& Elsner, M. (2020). Acquiring language from speech by learning to remember and predict. Proceedings of the 24th Conference on Computational Natural Language Learning, 195-214. https://doi.org/10.18653/v1/2020.conll-1.15

Singh, L., Cristia, A., Karasik, L. B., \& Oakes, L. (2021). Why are we still so WEIRD? Barriers and bridges towards a diversified science of early development. https://doi.org/10.31234/osf.io/ hgukc

Snoek, J., Rippel, O., Swersky, K., Kiros, R., Satish, N., Sundaram, N., Patwary, M. M. A., Prabhat, \& Adams, R. P. (2015). Scalable bayesian optimization using deep neural networks. Proceedings of the 32nd International Conference on Machine Learning (ICML). 
Soderstrom, M. (2007). Beyond babytalk: Re-evaluating the nature and content of speech input to preverbal infants. Developmental Review, 27(4), 501-532. https://doi.org/10.1016/j.dr. 2007.06.002

Sundara, M., \& Scutellaro, A. (2011). Rhythmic distance between languages affects the development of speech perception in bilingual infants. Journal of Phonetics, 39(4), 505-513. https://doi. org/10.1016/j.wocn.2010.08.006

Trainor, L. J., \& Desjardins, R. N. (2002). Pitch characteristics of infant-directed speech affect infants' ability to discriminate vowels. Psychonomic Bulletin and Review, 9(2), 335-340. https://doi.org/10.3758/BF03196290

Trehub, S. E. (1973). Infants' sensitivity to vowel and tonal contrasts. Developmental Psychology, 9(1), 91-96. https://doi.org/10.1037/h0034999

Trehub, S. E. (1976). The Discrimination of Foreign Speech Contrasts by Infants and Adults. Child Development, 47(2), 466. https://doi.org/10.2307/1128803

Tsuji, S., Bergmann, C., \& Cristia, A. (2014). Community-augmented meta-analyses: Toward cumulative data assessment. Perspectives on Psychological Science, 9(6), 661-665. https:// doi.org/10.1177/1745691614552498

Tsuji, S., \& Cristia, A. (2014). Perceptual attunement in vowels: A meta-analysis. Developmental Psychobiology, 56(2), 179-191. https://doi.org/10.1002/dev.21179

Vallabha, G. K., McClelland, J. L., Pons, F., Werker, J. F., \& Amano, S. (2007). Unsupervised learning of vowel categories from infant-directed speech. Proceedings of the National Academy of Sciences of the United States of America, 104 (33), 13273-13278. https://doi.org/10.1073/ pnas.0705369104

Versteegh, M., Thiollière, R., Schatz, T., Cao, X. N., Anguera, X., Jansen, A., \& Dupoux, E. (2015). The zero resource speech challenge 2015. Proceedings of the Annual Conference of the International Speech Communication Association (Interspeech), pp. 3169-3173. https://doi. org/10.21437/Interspeech.2015-638

Wang, H., Chen, Y., Lin, Y., Abesig, J., Wu, I. X., \& Tam, W. (2021). The methodological quality of individual participant data meta-analysis on intervention effects: Systematic review. The BMJ, 373. https://doi.org/10.1136/bmj.n736

Werker, J., \& Curtin, S. (2005). PRIMIR: A Developmental Framework of Infant Speech Processing. Language Learning and Development, 1(2), 197-234. https://doi.org/10.1080/15475441. 2005.9684216

Yarkoni, T. (2020). The generalizability crisis. Behavioral and Brain Sciences, 1-37. https://doi. org/10.1017/S0140525X20001685

Yarkoni, T., \& Westfall, J. (2017). Choosing prediction over explanation in psychology: Lessons from machine learning. Perspectives on Psychological Sciences, 12, 1100-1122. https://doi.org/ $10.1177 / 1745691617693393$

Yurovsky, D., \& Frank, M. C. (2015). An integrative account of constraints on cross-situational learning. Cognition, 145, 53-62. https://doi.org/10.1016/j.cognition.2015.07.013

Zhang, H., Zou, Y., \& Wang, H. (2021). Contrastive self-supervised learning for text-independent speaker verification. ICASSP 2021 - 2021 IEEE International Conference on Acoustics, Speech and Signal Processing (ICASSP), 6713-6717. https://doi.org/10.1109/ICASSP39728. 2021.9413351 


\section{Appendix A: Examples of effect size derivation from model responses}

As discussed in Section 3.3, a computational test for a model must convert model attentional preference scores $a_{i}(t)$ or model latent representations $\mathbf{z}_{i}(t)$ first into trial-specific responses $\alpha_{i}$ for each trial $v_{i}$, followed by calculation of the effect size using the measurements from all trials. The simplest approach to derive trial responses from $a_{i}(t)$ would be to aggregate the responses across time. For instance, if one trial corresponds to one stimulus condition of interest and the target variable is attentional preference towards the stimulus during that trial, the response $\alpha_{i}$ for trial $v_{i}$ could be extracted as the mean attentional preference towards the given stimulus:

$$
\alpha_{i}=\frac{1}{T_{i}} \sum_{t=1}^{T_{i}} a_{i}(t) .
$$

After extracting the responses for all trials, the standardized mean difference (Lipsey and Wilson, 2001) between conditions A and B could be calculated as

$$
d_{s, \text { model }}=\frac{\mu_{\alpha_{A}}-\mu_{\alpha_{B}}}{\sqrt{\frac{\left(n_{A}-1\right) \sigma_{\alpha_{A}}^{2}-\left(n_{B}-1\right) \sigma_{\alpha_{B}}^{2}}{n_{A}+n_{B}-2}}},
$$

where $\mu_{\alpha_{A}}$ and $\mu_{\alpha_{B}}$ are the mean responses for trials corresponding to conditions $\mathrm{A}$ and $\mathrm{B}$, respectively, while $\sigma_{\alpha_{A}}^{2}$ and $\sigma_{\alpha_{B}}^{2}$ are the corresponding variances, and $n_{A}$ and $n_{B}$ are the number of trials per condition. Note that this assumes that only one instance of the model is being tested, and that the test trials are mutually independent and identically distributed.

However, computational studies are often interested in discrimination between multiple stimuli without necessarily mapping the representations to overt attentional behavior through the preference scores $a(t)$. Besides enabling comparison against findings from brain recordings, this removes the need for a separate model mechanism for mapping the internal latent representations to the attentional scores, which is in itself a research problem that has not yet been much addressed in computational models of CLD. This gives rise to evaluation protocols that include comparison of representations within and across stimulus categories, such as comparing distances between vowel representations of the same vowel category and across vowel categories (cf., e.g., ABX-task in Schatz et al., 2013). This requires a mechanism for pairwise comparisons of latent representations $\mathbf{z}(t)$, which can be done by aggregation of difference scores $\alpha_{\text {diff }}$ between the latents from different conditions.

Potentially the simplest way to calculate $\alpha_{\text {diff }}$ would be to compare averages of $\mathbf{z}(t)$ for each stimulus pair of interest:

$$
\alpha_{i, \text { diff }}=\frac{1}{\left|T_{\mathrm{A}}\right|} \sum_{t_{1} \in T_{A}} \mathbf{z}_{i}\left(t_{1}\right)-\frac{1}{\left|T_{\mathrm{B}}\right|} \sum_{t_{2} \in T_{B}} \mathbf{z}_{i}\left(t_{2}\right),
$$

where $T_{\mathrm{A}}$ and $T_{\mathrm{B}}$ are the timesteps corresponding to stimulus $\mathrm{A}$ and $\mathrm{B}$ of trial $i$, respectively. However, this loses the temporal order and temporal fine detail of the representations. If the stimuli were always equal in duration, one could use Eq. (24) instead (using Euclidean distance as an example distance metric).

$$
\alpha_{i, \text { diff }}=\frac{1}{\left|T_{A}\right|} \sum_{t_{1} \in T_{A}, t_{2} \in T_{B}}\left\|\mathbf{z}_{i}\left(t_{1}\right)-\mathbf{z}_{i}\left(t_{2}\right)\right\|_{2}
$$

The same temporal difference is naturally applicable to attention preference scores as well, if one is interested in their temporal differences: 


$$
\alpha_{i, \text { diff }}=\frac{1}{\left|T_{\mathrm{A}}\right|} \sum_{t_{1} \in T_{\mathrm{A}}, t_{2} \in T_{\mathrm{B}}} a_{i}\left(t_{1}\right)-a_{i}\left(t_{2}\right)
$$

However, when dealing with real speech, stimulus durations are rarely identical. In this case, $\alpha_{\text {diff }}$ can be calculated with the help of dynamic time-warping (DTW; Sakoe and Chiba, 1978), which first performs temporal alignment of $\mathbf{z}\left(t_{1}\right) \rightarrow \hat{\mathbf{z}}\left(\hat{t_{1}}\right)$ and $\mathbf{z}\left(t_{2}\right) \rightarrow \hat{\mathbf{z}}\left(\hat{t_{2}}\right)$ such that the resulting corresponding representation durations $\left|\hat{T}_{\mathrm{A}}\right|=\left|\hat{T}_{\mathrm{B}}\right|$, and so that the total distance of the aligned vectors is minimized (preserving order and potentially constrained or penalized in terms of required frame repetitions/removals). Once aligned, one can apply Eq. (24) for the difference score (see Schatz et al., 2013). Again, the same steps are also applicable to $a(t)$ as needed.

Once the difference scores have been extracted for all trials in the experiment, effect size can be extracted, e.g., using Cohen's $d_{z}$ (Lakens, 2013):

$$
d_{z, \text { model }}=\frac{\mu_{\alpha_{\text {diff }}}}{\sqrt{\frac{\sum_{i}\left(\alpha_{i, \text { diff }}-\mu_{\alpha_{\text {diff }}}\right)^{2}}{N-1}}},
$$

where $\mu_{\alpha_{\text {diff }}}$ is the mean of difference scores $\alpha_{i \text {,diff }}$ across the full set of $N$ trials. If the test consists of multiple blocks of internally homogeneous stimuli (e.g., a set of synthetic speech trials and another set of natural speech trials), the resulting within-block correlations in model responses should ideally be taken into account in deriving the effect size (see, e.g., Lakens, 2013). In fact, we recommend to follow stimulus design of behavioral experiments, where differences in stimulus properties can be aligned with relevant moderators of interest (see Experiments for examples). As for studying model effect sizes as a function of the moderators $\beta$, one can simply extract the correct subsets of responses $\alpha_{i}$ where $\boldsymbol{\beta}_{i}$ has the desired values. Developmental trajectory testing is an exception to this, as the "age" of the model is based on the amount of training data seen by the model before the test, and thereby is independent of the test protocol itself. Hence, extraction of effect sizes as a function of model "age" cannot be managed inside the test.

In general, the exact procedure of deriving the condition-specific responses and resulting effect sizes for the model depends on the capability and test design in question. Hence, the procedures described are only meant as examples of how to get started with the conversion of model behavior into effects that could be compared to human data. 